\title{
Inequalities involving Dresher variance mean
}

\author{
Jiajin Wen ${ }^{1}$, Tianyong Han $^{1 *}$ and Sui Sun Cheng ${ }^{2}$
}

"Correspondence:
hantian123_123@163.com
${ }^{1}$ Institute of Mathematical
Inequalities and Applications,
Chengdu University, Chengdu,
Sichuan 610106, P.R. China
Full list of author information is
available at the end of the article

"Correspondence:

hantian123_123@163.com nequalities Chengdu University, Chengdu, Full list of author information is available at the end of the article

\begin{abstract}
Let $p$ be a real density function defined on a compact subset $\Omega$ of $\mathbb{R}^{m}$, and let $E(f, p)=\int_{\Omega} p f d \omega$ be the expectation of $f$ with respect to the density function $p$. In this paper, we define a one-parameter extension $\operatorname{Var}_{\gamma}(f, p)$ of the usual variance $\operatorname{Var}(f, p)=E\left(f^{2}, p\right)-E^{2}(f, p)$ of a positive continuous function $f$ defined on $\Omega$. By means of this extension, a two-parameter mean $V_{r, s}(f, p)$, called the Dresher variance mean, is then defined. Their properties are then discussed. In particular, we establish a Dresher variance mean inequality $\min _{t \in \Omega}\{f(t)\} \leq V_{r, s}(f, p) \leq \max _{t \in \Omega}\{f(t)\}$, that is to say, the Dresher variance mean $V_{r, s}(f, p)$ is a true mean of $f$. We also establish a Dresher-type inequality $V_{r, s}(f, p) \geq V_{r^{*}, s^{*}}(f, p)$ under appropriate conditions on $r, s, r^{*}, s^{*}$; and finally, a $V$-E inequality $V_{r, s}(f, p) \geq\left(\frac{s}{r}\right)^{1 /(r-s)} E(f, p)$ that shows that $V_{r, s}(f, p)$ can be compared with $E(f, p)$. We are also able to illustrate the uses of these results in space science.
\end{abstract}

MSC: 26D15; 26E60; 62J10

Keywords: power mean; Dresher mean; $\boldsymbol{\gamma}$-variance; Dresher variance mean; Dresher inequality; Jensen inequality; $\lambda$-gravity function

\section{Introduction and main results}

As indicated in the monograph [1], the concept of mean is basic in the theory of inequalities and its applications. Indeed, there are many inequalities involving different types of mean in [1-18], and a great number of them have been used in mathematics and other natural sciences.

Dresher in [14], by means of moment space techniques, proved the following inequality: If $\rho \geq 1 \geq \sigma \geq 0, f, g \geq 0$, and $\phi$ is a distribution function, then

$$
\left[\frac{\int(f+g)^{\rho} d \phi}{\int(f+g)^{\sigma} d \phi}\right]^{1 /(\rho-\sigma)} \leq\left(\frac{\int f^{\rho} d \phi}{\int f^{\sigma} d \phi}\right)^{1 /(\rho-\sigma)}+\left(\frac{\int g^{\rho} d \phi}{\int g^{\sigma} d \phi}\right)^{1 /(\rho-\sigma)}
$$

This result is referred to as Dresher's inequality by Daskin [15], Beckenbach and Bellman [16] ( $\$ 24$ in Ch. 1) and Hu [18] (p.21). Note that if we define

$$
\bar{E}(f, \phi)=\int f d \phi,
$$

and

$$
D_{r, s}(f, \phi)=\left(\frac{\bar{E}\left(f^{r}, \phi\right)}{\bar{E}\left(f^{s}, \phi\right)}\right)^{1 /(r-s)}, \quad r, s \in \mathbb{R}
$$

o 2013 Wen et al.; licensee Springer. This is an Open Access article distributed under the terms of the Creative Commons Attribution License (http://creativecommons.org/licenses/by/2.0), which permits unrestricted use, distribution, and reproduction in any medium, provided the original work is properly cited. 
then the above inequality can be rewritten as

$$
D_{\rho, \sigma}(f+g, \phi) \leq D_{\rho, \sigma}(f, \phi)+D_{\rho, \sigma}(g, \phi) .
$$

$D_{r, s}(f, \phi)$ is the well-known Dresher mean of the function $f$ (see $[10,11,14-18]$ ), which involves two parameters $r$ and $s$ and has applications in the theory of probability.

However, variance is also a crucial quantity in probability and statistics theory. It is, therefore, of interest to establish inequalities for various variances as well. In this paper, we introduce generalized 'variances' and establish several inequalities involving them. Although we may start out under a more general setting, for the sake of simplicity, we choose to consider the variance of a continuous function $f$ with respect to a weight function $p$ (including probability densities) defined on a closed and bounded domain $\Omega$ in $\mathbb{R}^{m}$ instead of a distribution function.

More precisely, unless stated otherwise, in all later discussions, let $\Omega$ be a fixed, nonempty, closed and bounded domain in $\mathbb{R}^{m}$ and let $p: \Omega \rightarrow(0, \infty)$ be a fixed function which satisfies $\int_{\Omega} p d \omega=1$. For any continuous function $f: \Omega \rightarrow \mathbb{R}$, we write

$$
E(f, p)=\int_{\Omega} p f d \omega
$$

which may be regarded as the weighted mean of the function $f$ with respect to the weight function $p$.

Recall that the standard variance (see [12] and [19]) of a random variable $f$ with respect to a density function $p$ is

$$
\operatorname{Var}(f, p)=E\left(f^{2}, p\right)-E^{2}(f, p) .
$$

We may, however, generalize this to the $\gamma$-variance of the function $f: \Omega \rightarrow(0, \infty)$ defined by

$$
\operatorname{Var}_{\gamma}(f, p)= \begin{cases}\frac{2}{\gamma(\gamma-1)}\left[E\left(f^{\gamma}, p\right)-E^{\gamma}(f, p)\right], & \gamma \neq 0,1, \\ 2[\ln E(f, p)-E(\ln f, p)], & \gamma=0, \\ 2[E(f \ln f, p)-E(f, p) \ln E(f, p)], & \gamma=1 .\end{cases}
$$

According to this definition, we know that $\gamma$-variance $\operatorname{Var}_{\gamma}(f, p)$ is a functional of the function $f: \Omega \rightarrow(0, \infty)$ and $p: \Omega \rightarrow(0, \infty)$, and such a definition is compatible with the generalized integral means studied elsewhere (see, e.g., [1-6]). Indeed, according to the power mean inequality (see, e.g., [1-9]), we may see that

$$
\operatorname{Var}_{\gamma}(f, p) \geq 0, \quad \forall \gamma \in \mathbb{R} .
$$

Let $f: \Omega \rightarrow(0, \infty)$ and $g: \Omega \rightarrow(0, \infty)$ be two continuous functions. We define

$$
\operatorname{Cov}_{\gamma}(f, g)=E\left\{\left[f^{\gamma}-E^{\gamma}(f, p)\right] \circ\left[g^{\gamma}-E^{\gamma}(g, p)\right], p\right\}
$$


is the $\gamma$-covariance of the function $f: \Omega \rightarrow(0, \infty)$ and the function $g: \Omega \rightarrow(0, \infty)$, where $\gamma \in \mathbb{R}$ and the function $a \circ b: \mathbb{R}^{2} \rightarrow \mathbb{R}$ is defined as follows:

$$
a \circ b= \begin{cases}\sqrt{a b}, & a \neq b, a b \geq 0, \\ -\sqrt{-a b}, & a \neq b, a b<0, \\ a, & a=b .\end{cases}
$$

According to this definition, we get

$$
\operatorname{Cov}_{0}(f, g) \equiv 0, \quad \operatorname{Cov}_{1}(f, f) \equiv 0
$$

and

$$
\operatorname{Var}_{\gamma}(f, p)= \begin{cases}\frac{2}{\gamma(\gamma-1)} \operatorname{Cov}_{\gamma}(f, f), & \gamma \neq 0,1, \\ \lim _{\gamma \rightarrow 0} \frac{2}{\gamma(\gamma-1)} \operatorname{Cov}_{\gamma}(f, f), & \gamma=0, \\ \lim _{\gamma \rightarrow 1} \frac{2}{\gamma(\gamma-1)} \operatorname{Cov}_{\gamma}(f, f), & \gamma=1 .\end{cases}
$$

If we define

$$
\operatorname{Abscov}_{\gamma}(f, g)=E\left\{\left|\left[f^{\gamma}-E^{\gamma}(f, p)\right] \circ\left[g^{\gamma}-E^{\gamma}(g, p)\right]\right|, p\right\}
$$

is the $\gamma$-absolute covariance of the function $f: \Omega \rightarrow(0, \infty)$ and the function $g: \Omega \rightarrow$ $(0, \infty)$, then we have that

$$
\left|\frac{\operatorname{Cov}_{\gamma}(f, g)}{\sqrt{\operatorname{Abscov}_{\gamma}(f, f)} \sqrt{\operatorname{Abscov}_{\gamma}(g, g)}}\right| \leq 1
$$

for $\gamma \neq 0$ by the Cauchy inequality

$$
\left|\int_{\Omega} p f g d \omega\right| \leq \sqrt{\int_{\Omega} p f^{2} d \omega} \sqrt{\int_{\Omega} p g^{2} d \omega .}
$$

Therefore, we can define the $\gamma$-correlation coefficient of the function $f: \Omega \rightarrow(0, \infty)$ and the function $g: \Omega \rightarrow(0, \infty)$ as follows:

$$
\rho_{\gamma}(f, g)= \begin{cases}\frac{\operatorname{Cov}_{\gamma}(f, g)}{\sqrt{\operatorname{Abscov}_{\gamma}(f, f)} \sqrt{\operatorname{Abscov}_{\gamma}(g, g)}}, & \gamma \neq 0, \\ \lim _{\gamma \rightarrow 0} \frac{\operatorname{Cov}_{\gamma}(f, g)}{\sqrt{\operatorname{Abscov}_{\gamma}(f, f)} \sqrt{\operatorname{Abscov}_{\gamma}(g, g)}}, & \gamma=0,\end{cases}
$$

where $\rho_{\gamma}(f, g) \in[-1,1]$.

By means of $\operatorname{Var}_{\gamma}(f, p)$, we may then define another two-parameter mean. This new twoparameter mean $V_{r, s}(f, p)$ will be called the Dresher variance mean of the function $f$. It is motivated by (1) and $[10,11,14-18]$ and is defined as follows. Given $(r, s) \in \mathbb{R}^{2}$ and the continuous function $f: \Omega \rightarrow(0, \infty)$. If $f$ is a constant function defined by $f(x)=c$ for any $x \in \Omega$, we define the functional

$$
V_{r, s}(f, p)=c,
$$


and if $f$ is not a constant function, we define the functional

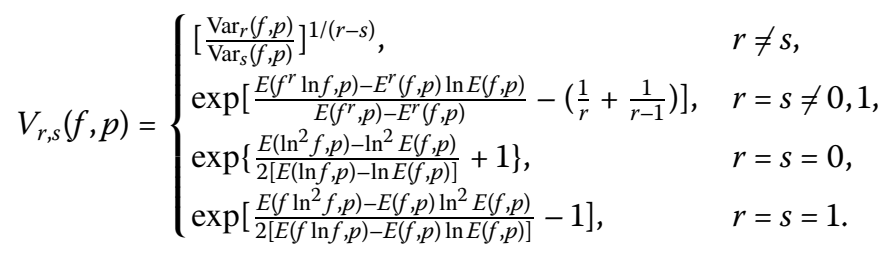

Since the function $f: \Omega \rightarrow(0, \infty)$ is continuous, we know that the functions $p f^{\gamma}$ and $p f^{\gamma} \ln f$ are integrable for any $\gamma \in \mathbb{R}$. Thus $\operatorname{Var}_{\gamma}(f, p)$ and $V_{r, s}(f, p)$ are well defined. Since

$$
\begin{aligned}
& \lim _{\gamma \rightarrow \gamma^{*}} \operatorname{Var}_{\gamma}(f, p)=\operatorname{Var}_{\gamma^{*}}(f, p), \\
& \lim _{(r, s) \rightarrow\left(r^{*}, s^{*}\right)} V_{r, s}(f, p)=V_{r^{*}, s^{*}}(f, p),
\end{aligned}
$$

$\operatorname{Var}_{\gamma}(f, p)$ is continuous with respect to $\gamma \in \mathbb{R}$ and $V_{r, s}(f, p)$ continuous with respect to $(r, s) \in \mathbb{R}^{2}$.

We will explain why we are concerned with our one-parameter variance $\operatorname{Var}_{\gamma}(f, p)$ and the two-parameter mean $V_{r, s}(f, p)$ by illustrating their uses in statistics and space science.

Before doing so, we first state three main theorems of our investigations.

Theorem 1 (Dresher variance mean inequality) For any continuous function $f: \Omega \rightarrow$ $(0, \infty)$, we have

$$
\min _{t \in \Omega}\{f(t)\} \leq V_{r, s}(f, p) \leq \max _{t \in \Omega}\{f(t)\} .
$$

Theorem 2 (Dresher-type inequality) Let the function $f: \Omega \rightarrow(0, \infty)$ be continuous. If $(r, s) \in \mathbb{R}^{2},\left(r^{*}, s^{*}\right) \in \mathbb{R}^{2}, \max \{r, s\} \geq \max \left\{r^{*}, s^{*}\right\}$ and $\min \{r, s\} \geq \min \left\{r^{*}, s^{*}\right\}$, then

$$
V_{r, s}(f, p) \geq V_{r^{*}, s^{*}}(f, p)
$$

Theorem 3 ( $V$-E inequality) For any continuous function $f: \Omega \rightarrow(0, \infty)$, we have

$$
V_{r, s}(f, p) \geq\left(\frac{s}{r}\right)^{\frac{1}{r-s}} E(f, p)
$$

moreover, the coefficient $\left(\frac{s}{r}\right)^{\frac{1}{r-s}}$ in (4) is the best constant.

From Theorem 1, we know that $V_{r, s}(f, p)$ is a certain mean value of $f$. Theorem 2 is similar to the well-known Dresher inequality stated in Lemma 3 below (see, e.g., [2], p.74 and $[10,11])$. By Theorem 2 , we see that $V_{r, s}(f, p)$ and $V_{r^{*}, s^{*}}(f, p)$ can be compared under appropriate conditions on $r, s, r^{*}, s^{*}$. Theorem 3 states a connection of $V_{r, s}(f, p)$ with the weighted mean $E(f, p)$.

Let $\Omega$ be a fixed, nonempty, closed and bounded domain in $\mathbb{R}^{m}$, and let $p=p(X)$ be the density function with support in $\Omega$ for the random vector $X=\left(x_{1}, x_{2}, \ldots, x_{m}\right)$. For any function $f: \Omega \rightarrow(0, \infty)$, the mean of the random variable $f(X)$ is

$$
E[f(X)]=\int_{\Omega} p f d \omega=E(f, p)
$$


moreover, its variance is

$$
\operatorname{Var}[f(X)]=E\left[f^{2}(X)\right]-E^{2}[f(X)]=\int_{\Omega} p\{f-E[f(X)]\}^{2} d \omega=\operatorname{Var}_{2}(f, p)
$$

Therefore,

$$
\operatorname{Var}_{\gamma}[f(X)]=\operatorname{Var}_{\gamma}(f, p), \quad \gamma \in \mathbb{R}
$$

may be regarded as a $\gamma$-variance and

$$
V_{r, s}[f(X)]=V_{r, s}(f, p), \quad(r, s) \in \mathbb{R}^{2}
$$

may be regarded as a Dresher variance mean of the random variable $f(X)$.

Note that by Theorem 1 , we have

$$
\min _{X \in \Omega}\{f(X)\} \leq V_{r, s}[f(X)] \leq \max _{X \in \Omega}\{f(X)\}, \quad(r, s) \in \mathbb{R}^{2} ;
$$

and by Theorem 2 , we see that for any $r, s, r^{*}, s^{*} \in \mathbb{R}$ such that

$$
\max \{r, s\} \geq \max \left\{r^{*}, s^{*}\right\} \quad \text { and } \min \{r, s\} \geq \min \left\{r^{*}, s^{*}\right\},
$$

then

$$
V_{r, s}[f(X)] \geq V_{r^{*}, s^{*}}[f(X)]
$$

and by Theorem 3 , if $r>s \geq 1$, then

$$
\frac{\operatorname{Var}_{r}[f(X)]}{\operatorname{Var}_{s}[f(X)]} \geq \frac{s}{r} E^{r-s}[f(X)],
$$

where the coefficient $s / r$ is the best constant.

In the above results, $\Omega$ is a closed and bounded domain of $\mathbb{R}^{m}$. However, we remark that our results still hold if $\Omega$ is an unbounded domain of $\mathbb{R}^{m}$ or some values of $f$ are 0 , as long as the integrals in Theorems 1-3 are convergent. Such extended results can be obtained by standard techniques in real analysis by applying continuity arguments and Lebesgue's dominated convergence theorem, and hence we need not spell out all the details in this paper.

\section{Proof of Theorem 1}

For the sake of simplicity, we employ the following notations. Let $n$ be an integer greater than or equal to 2 , and let $N_{n}=\{1,2, \ldots, n\}$. For real $n$-vectors $x=\left(x_{1}, \ldots, x_{n}\right)$ and $p=$ $\left(p_{1}, \ldots, p_{n}\right)$, the dot product of $p$ and $x$ is denoted by $A(x, p)=p \cdot x=\sum_{i=1}^{n} p_{i} x_{i}$, where $p \in S^{n}$ and $S^{n}=\left\{p \in[0, \infty)^{n} \mid \sum_{i=1}^{n} p_{i}=1\right\}, S^{n}$ is an $(n-1)$-dimensional simplex. If $\phi$ is a real function of a real variable, for the sake of convenience, we set the vector function

$$
\phi(x)=\left(\phi\left(x_{1}\right), \phi\left(x_{2}\right), \ldots, \phi\left(x_{n}\right)\right) .
$$


Suppose that $p \in S^{n}$ and $\gamma, r, s \in \mathbb{R}$. If $x \in(0, \infty)^{n}$, then

$$
\operatorname{Var}_{\gamma}(x, p)= \begin{cases}\frac{2}{\gamma(\gamma-1)}\left[A\left(x^{\gamma}, p\right)-A^{\gamma}(x, p)\right], & \gamma \neq 0,1, \\ 2[\ln A(x, p)-A(\ln x, p)], & \gamma=0, \\ 2[A(x \ln x, p)-A(x, p) \ln A(x, p)], & \gamma=1\end{cases}
$$

is called the $\gamma$-variance of the vector $x$ with respect to $p$. If $x \in(0, \infty)^{n}$ is a constant $n$ vector, then we define

$$
V_{r, s}(x, p)=x_{1}
$$

while if $x$ is not a constant vector (i.e., there exist $i, j \in N_{n}$ such that $x_{i} \neq x_{j}$ ), then we define

$$
V_{r, s}(x, p)= \begin{cases}{\left[\frac{\operatorname{Var}_{r}(x, p)}{\operatorname{Var}_{s}(x, p)}\right] \frac{1}{r-s},} & r \neq s, \\ \exp \left[\frac{A\left(x^{r} \ln x, p\right)-A^{r}(x, p) \ln A(x, p)}{A\left(x^{r}, p\right)-A^{r}(x, p)}-\left(\frac{1}{r}+\frac{1}{r-1}\right)\right], & r=s \neq 0,1, \\ \exp \left\{\frac{A\left(\ln ^{2} x, p\right)-\ln ^{2} A(x, p)}{2[A(\ln x, p)-\ln A(x, p)]}+1\right\}, & r=s=0, \\ \exp \left\{\frac{A\left(x \ln \ln ^{2} x, p\right)-A(x, p) \ln { }^{2} A(x, p)}{2[A(x \ln x, p)-A(x, p) \ln A(x, p)]}-1\right\}, & r=s=1 .\end{cases}
$$

$V_{r, s}(x, p)$ is called the Dresher variance mean of the vector $x$.

Clearly, $\operatorname{Var}_{\gamma}(x, p)$ is nonnegative and is continuous with respect to $\gamma \in \mathbb{R}$. $V_{r, s}(x, p)=$ $V_{s, r}(x, p)$ and is also continuous with respect to $(r, s)$ in $\mathbb{R}^{2}$.

Lemma 1 Let I be a real interval. Suppose the function $\phi: I \rightarrow \mathbb{R}$ is $C^{(2)}$, i.e., twice continuously differentiable. If $x \in I^{n}$ and $p \in S^{n}$, then

$$
A(\phi(x), p)-\phi(A(x, p))=\sum_{1 \leq i<j \leq n} p_{i} p_{j}\left\{\iint_{\Phi} \phi^{\prime \prime}\left[w_{i, j}\left(x, p, t_{1}, t_{2}\right)\right] d t_{1} d t_{2}\right\}\left(x_{i}-x_{j}\right)^{2}
$$

where $\Phi$ is the triangle $\left\{\left(t_{1}, t_{2}\right) \in[0, \infty)^{2} \mid t_{1}+t_{2} \leq 1\right\}$ and

$$
w_{i, j}\left(x, p, t_{1}, t_{2}\right)=t_{1} x_{i}+t_{2} x_{j}+\left(1-t_{1}-t_{2}\right) A(x, p) .
$$

Proof Note that

$$
\begin{aligned}
& \iint_{\Phi} \phi^{\prime \prime}\left[w_{i, j}\left(x, p, t_{1}, t_{2}\right)\right] d t_{1} d t_{2} \\
& =\int_{0}^{1} d t_{1} \int_{0}^{1-t_{1}} \phi^{\prime \prime}\left[w_{i, j}\left(x, p, t_{1}, t_{2}\right)\right] d t_{2} \\
& =\frac{1}{x_{j}-A(x, p)} \int_{0}^{1} d t_{1} \int_{0}^{1-t_{1}} \phi^{\prime \prime}\left[w_{i, j}\left(x, p, t_{1}, t_{2}\right)\right] d\left[w_{i, j}\left(x, p, t_{1}, t_{2}\right)\right] \\
& =\left.\frac{1}{x_{j}-A(x, p)} \int_{0}^{1} d t_{1} \phi^{\prime}\left[t_{1} x_{i}+t_{2} x_{j}+\left(1-t_{1}-t_{2}\right) A(x, p)\right]\right|_{0} ^{1-t_{1}} \\
& =\frac{1}{x_{j}-A(x, p)} \int_{0}^{1}\left\{\phi^{\prime}\left[t_{1} x_{i}+\left(1-t_{1}\right) x_{j}\right]-\phi^{\prime}\left[t_{1} x_{i}+\left(1-t_{1}\right) A(x, p)\right]\right\} d t_{1}
\end{aligned}
$$




$$
\begin{aligned}
& =\left.\frac{1}{x_{j}-A(x, p)}\left\{\frac{\phi\left[t_{1} x_{i}+\left(1-t_{1}\right) x_{j}\right]}{x_{i}-x_{j}}-\frac{\phi\left[t_{1} x_{i}+\left(1-t_{1}\right) A(x, p)\right]}{x_{i}-A(x, p)}\right\}\right|_{0} ^{1} \\
& =\frac{1}{x_{j}-A(x, p)}\left[\frac{\phi\left(x_{i}\right)-f\left(x_{j}\right)}{x_{i}-x_{j}}-\frac{\phi\left(x_{i}\right)-\phi(A(x, p))}{x_{i}-A(x, p)}\right] \\
& =\frac{1}{\left(x_{i}-x_{j}\right)\left[x_{j}-A(x, p)\right]\left[x_{i}-A(x, p)\right]}\left|\begin{array}{ccc}
\phi(A(x, p)) & A(x, p) & 1 \\
\phi\left(x_{i}\right) & x_{i} & 1 \\
\phi\left(x_{j}\right) & x_{j} & 1
\end{array}\right| .
\end{aligned}
$$

Hence,

$$
\begin{aligned}
& \sum_{1 \leq i<j \leq n} p_{i} p_{j}\left\{\iint_{\Phi} \phi^{\prime \prime}\left[t_{1} x_{i}+t_{2} x_{j}+\left(1-t_{1}-t_{2}\right) A(x, p)\right] d t_{1} d t_{2}\right\}\left(x_{i}-x_{j}\right)^{2} \\
& =\sum_{1 \leq i<j \leq n} p_{i} p_{j} \frac{x_{i}-x_{j}}{\left[x_{j}-A(x, p)\right]\left[x_{i}-A(x, p)\right]}\left|\begin{array}{ccc}
\phi(A(x, p)) & A(x, p) & 1 \\
\phi\left(x_{i}\right) & x_{i} & 1 \\
\phi\left(x_{j}\right) & x_{j} & 1
\end{array}\right| \\
& =\frac{1}{2} \sum_{1 \leq i, j \leq n} p_{i} p_{j}\left[\frac{1}{x_{j}-A(x, p)}-\frac{1}{x_{i}-A(x, p)}\right]\left|\begin{array}{ccc}
\phi(A(x, p)) & A(x, p) & 1 \\
\phi\left(x_{i}\right) & x_{i} & 1 \\
\phi\left(x_{j}\right) & x_{j} & 1
\end{array}\right| \\
& =\frac{1}{2}\left[\sum_{1 \leq i, j \leq n} p_{i} p_{j} \frac{1}{x_{j}-A(x, p)}\left|\begin{array}{ccc}
\phi(A(x, p)) & A(x, p) & 1 \\
\phi\left(x_{i}\right) & x_{i} & 1 \\
\phi\left(x_{j}\right) & x_{j} & 1
\end{array}\right|\right. \\
& \left.-\sum_{1 \leq i, j \leq n} p_{i} p_{j} \frac{1}{x_{i}-A(x, p)}\left|\begin{array}{ccc}
\phi(A(x, p)) & A(x, p) & 1 \\
\phi\left(x_{i}\right) & x_{i} & 1 \\
\phi\left(x_{j}\right) & x_{j} & 1
\end{array}\right|\right] \\
& =\frac{1}{2}\left[\sum_{j=1}^{n} \frac{p_{j}}{x_{j}-A(x, p)} \sum_{i=1}^{n} \begin{array}{ccc}
\phi(A(x, p)) & A(x, p) & 1 \\
p_{i} \phi\left(x_{i}\right) & p_{i} x_{i} & p_{i} \\
\phi\left(x_{j}\right) & x_{j} & 1
\end{array} \mid\right. \\
& \left.-\sum_{i=1}^{n} \frac{p_{i}}{x_{i}-A(x, p)} \sum_{j=1}^{n}\left|\begin{array}{ccc}
\phi(A(x, p)) & A(x, p) & 1 \\
\phi\left(x_{i}\right) & x_{i} & 1 \\
p_{j} \phi\left(x_{j}\right) & p_{j} x_{j} & p_{j}
\end{array}\right|\right] \\
& =\frac{1}{2}\left[\sum_{j=1}^{n} \frac{p_{j}}{x_{j}-A(x, p)}\left|\begin{array}{ccc}
\phi(A(x, p)) & A(x, p) & 1 \\
\sum_{i=1}^{n} p_{i} \phi\left(x_{i}\right) & \sum_{i=1}^{n} p_{i} x_{i} & \sum_{i=1}^{n} p_{i} \\
\phi\left(x_{j}\right) & x_{j} & 1
\end{array}\right|\right. \\
& \left.-\sum_{i=1}^{n} \frac{p_{i}}{x_{i}-A(x, p)}\left|\begin{array}{ccc}
\phi(A(x, p)) & A(x, p) & 1 \\
\phi\left(x_{i}\right) & x_{i} & 1 \\
\sum_{j=1}^{n} p_{j} \phi\left(x_{j}\right) & \sum_{j=1}^{n} p_{j} x_{j} & \sum_{j=1}^{n} p_{j}
\end{array}\right|\right] \\
& =\frac{1}{2}\left[\sum_{j=1}^{n} \frac{p_{j}}{x_{j}-A(x, p)}\left|\begin{array}{ccc}
\phi(A(x, p)) & A(x, p) & 1 \\
A(\phi(x), p) & A(x, p) & 1 \\
\phi\left(x_{j}\right) & x_{j} & 1
\end{array}\right|\right.
\end{aligned}
$$




$$
\begin{aligned}
& \left.-\sum_{i=1}^{n} \frac{p_{i}}{x_{i}-A(x, p)} \mid \begin{array}{ccc}
\phi(A(x, p)) & A(x, p) & 1 \\
\phi\left(x_{i}\right) & x_{i} & 1 \\
A(\phi(x), p) & A(x, p) & 1
\end{array}\right] \\
& =\frac{1}{2}\left[\sum_{j=1}^{n} \frac{p_{j}}{x_{j}-A(x, p)}\left|\begin{array}{ccc}
\phi(A(x, p))-A(\phi(x), p) & 0 & 0 \\
A(\phi(x), p) & A(x, p) & 1 \\
\phi\left(x_{j}\right) & x_{j} & 1
\end{array}\right|\right. \\
& \left.-\sum_{i=1}^{n} \frac{p_{i}}{x_{i}-A(x, p)}\left|\begin{array}{ccc}
\phi(A(x, p))-A(\phi(x), p) & 0 & 0 \\
\phi\left(x_{i}\right) & x_{i} & 1 \\
A(\phi(x), p) & A(x, p) & 1
\end{array}\right|\right] \\
& =\frac{1}{2}\left\{\sum_{j=1}^{n} \frac{p_{j}}{x_{j}-A(x, p)}[\phi(A(x, p))-A(\phi(x), p)]\left[A(x, p)-x_{j}\right]\right. \\
& \left.-\sum_{i=1}^{n} \frac{p_{i}}{x_{i}-A(x, p)}[\phi(A(x, p))-A(\phi(x), p)]\left[x_{i}-A(x, p)\right]\right\} \\
& =\frac{1}{2}\left\{\sum_{j=1}^{n} p_{j}[A(\phi(x), p)-\phi(A(x, p))]+\sum_{i=1}^{n} p_{i}[A(\phi(x), p)-\phi(A(x, p))]\right\} \\
& =A(\phi(x), p)-\phi(A(x, p)) \text {. }
\end{aligned}
$$

Therefore, (8) holds. The proof is complete.

Remark 1 The well-known Jensen inequality can be described as follows [20-22]: If the function $\phi: I \rightarrow \mathbb{R}$ satisfies $\phi^{\prime \prime}(t) \geq 0$ for all $t$ in the interval $I$, then for any $x \in I^{n}$ and $p \in S^{n}$, we have

$$
A(\phi(x), p) \geq \phi(A(x, p)) .
$$

The above proof may be regarded as a constructive proof of (9).

Remark 2 We remark that the Dresher variance mean $V_{r, s}(x, p)$ extends the variance mean $V_{r, 2}(x, p)$ (see [2], p.664, and [13, 21]), and Lemma 1 is a generalization of (2.23) of [19].

Lemma 2 If $x \in(0, \infty)^{n}, p \in S^{n}$ and $(r, s) \in \mathbb{R}^{2}$, then

$$
\min \{x\}=\min \left\{x_{1}, \ldots, x_{n}\right\} \leq V_{r, s}(x, p) \leq \max \left\{x_{1}, \ldots, x_{n}\right\}=\max \{x\} .
$$

Proof If $x$ is a constant vector, our assertion is clearly true. Let $x$ be a non-constant vector, that is, there exist $i, j \in N_{n}$ such that $x_{i} \neq x_{j}$. Note that $V_{r, s}(x, p)=V_{s, r}(x, p)$ and $V_{r, s}(x, p)$ is continuous with respect to $(r, s)$ in $\mathbb{R}^{2}$, we may then assume that

$$
r(r-1) s(s-1) \neq 0 \quad \text { and } \quad r-s>0 .
$$

In (8), let $\phi:(0, \infty) \rightarrow \mathbb{R}$ be defined by $\phi(t)=t^{\gamma}$, where $\gamma(\gamma-1) \neq 0$. Then we obtain

$$
\operatorname{Var}_{\gamma}(x, p)=2 \sum_{1 \leq i<j \leq n} p_{i} p_{j}\left\{\iint_{\Phi}\left[w_{i, j}\left(x, p, t_{1}, t_{2}\right)\right]^{\gamma-2} d t_{1} d t_{2}\right\}\left(x_{i}-x_{j}\right)^{2} .
$$


Since

$$
\min \{x\}=\min \left\{x_{1}, \ldots, x_{n}\right\} \leq w_{i, j}\left(x, p, t_{1}, t_{2}\right) \leq \max \left\{x_{1}, \ldots, x_{n}\right\}=\max \{x\}
$$

by (11), (12), $r-s>0$ and the fact that

$$
\begin{aligned}
V_{r, s}(x, p) \\
\quad=\left[\frac{\operatorname{Var}_{r}(x, p)}{\operatorname{Var}_{s}(x)}\right]^{\frac{1}{r-s}} \\
=\left[\frac{\sum_{1 \leq i<j \leq n} p_{i} p_{j}\left(x_{i}-x_{j}\right)^{2} \iint_{\Phi} w_{i, j}^{r-2}\left(x, p, t_{1}, t_{2}\right) d t_{1} d t_{2}}{\sum_{1 \leq i<j \leq n} p_{i} p_{j}\left(x_{i}-x_{j}\right)^{2} \iint_{\Phi} w_{i, j}^{s-2}\left(x, p, t_{1}, t_{2}\right) d t_{1} d t_{2}}\right]^{\frac{1}{r-s}} \\
=\left[\frac{\sum_{1 \leq i<j \leq n} p_{i} p_{j}\left(x_{i}-x_{j}\right)^{2} \iint_{\Phi} w_{i, j}^{s-2}\left(x, p, t_{1}, t_{2}\right) \times w_{i, j}^{r-s}\left(x, p, t_{1}, t_{2}\right) d t_{1} d t_{2}}{\sum_{1 \leq i<j \leq n} p_{i} p_{j}\left(x_{i}-x_{j}\right)^{2} \iint_{\Phi} w_{i, j}^{s-2}\left(x, p, t_{1}, t_{2}\right) d t_{1} d t_{2}}\right]^{\frac{1}{r-s}},
\end{aligned}
$$

we obtain (10). This concludes the proof.

We may now turn to the proof of Theorem 1.

Proof First, we may assume that $f$ is a nonconstant function and that

$$
r(r-1) s(s-1) \neq 0, \quad r-s>0 .
$$

Let

$$
T=\left\{\Delta \Omega_{1}, \Delta \Omega_{2}, \ldots, \Delta \Omega_{n}\right\}
$$

be a partition of $\Omega$, and let

$$
\|T\|=\max _{1 \leq i \leq n} \max _{X, Y \in \Delta \Omega_{i}}\{\|X-Y\|\}
$$

be the 'norm' of the partition $T$, where

$$
\|X-Y\|=\sqrt{(X-Y) \cdot(X-Y)}
$$

is the length of the vector $X-Y$. Pick any $\xi_{i} \in \Delta \Omega_{i}$ for each $i=1,2, \ldots, n$, set

$$
\xi=\left(\xi_{1}, \xi_{2}, \ldots, \xi_{n}\right), \quad f(\xi)=\left(f\left(\xi_{1}\right), f\left(\xi_{2}\right), \ldots, f\left(\xi_{n}\right)\right),
$$

and

$$
\bar{p}(\xi)=\left(\bar{p}_{1}(\xi), \bar{p}_{2}(\xi), \ldots, \bar{p}_{n}(\xi)\right)=\frac{\left(p\left(\xi_{1}\right)\left|\Delta \Omega_{1}\right|, p\left(\xi_{2}\right)\left|\Delta \Omega_{2}\right|, \ldots, p\left(\xi_{n}\right)\left|\Delta \Omega_{n}\right|\right)}{\sum_{i=1}^{n} p\left(\xi_{i}\right)\left|\Delta \Omega_{i}\right|},
$$

then

$$
\lim _{\|T\| \rightarrow 0} \sum_{i=1}^{n} p\left(\xi_{i}\right)\left|\Delta \Omega_{i}\right|=\int_{\Omega} p d \omega=1
$$

where $\left|\Delta \Omega_{i}\right|$ is the $m$-dimensional volume of $\Delta \Omega_{i}$ for $i=1,2, \ldots, n$. 
Furthermore, when $\gamma(\gamma-1) \neq 0$, we have

$$
\begin{aligned}
\operatorname{Var}_{\gamma}(f, p)= & \frac{2}{\gamma(\gamma-1)}\left[\lim _{\|T\| \rightarrow 0} \sum_{i=1}^{n} p\left(\xi_{i}\right) f^{\gamma}\left(\xi_{i}\right)\left|\Delta \Omega_{i}\right|-\left(\lim _{\|T\| \rightarrow 0} \sum_{i=1}^{n} p\left(\xi_{i}\right) f\left(\xi_{i}\right)\left|\Delta \Omega_{i}\right|\right)^{\gamma}\right] \\
= & \frac{2}{\gamma(\gamma-1)}\left[\left(\lim _{\|T\| \rightarrow 0} \sum_{i=1}^{n} p\left(\xi_{i}\right)\left|\Delta \Omega_{i}\right|\right)\left(\lim _{\|T\| \rightarrow 0} \sum_{i=1}^{n} \bar{p}_{i}(\xi) f^{\gamma}\left(\xi_{i}\right)\right)\right. \\
& \left.-\left(\lim _{\|T\| \rightarrow 0} \sum_{i=1}^{n} p\left(\xi_{i}\right)\left|\Delta \Omega_{i}\right|\right)^{\gamma}\left(\lim _{\|T\| \rightarrow 0} \sum_{i=1}^{n} \bar{p}_{i}(\xi) f\left(\xi_{i}\right)\right)^{\gamma}\right] \\
= & \frac{2}{\gamma(\gamma-1)}\left[\lim _{\|T\| \rightarrow 0} \sum_{i=1}^{n} \bar{p}_{i}(\xi) f^{\gamma}\left(\xi_{i}\right)-\left(\lim _{\|T\| \rightarrow 0} \sum_{i=1}^{n} \bar{p}_{i}(\xi) f\left(\xi_{i}\right)\right)^{\gamma}\right] \\
= & \lim _{\|T\| \rightarrow 0} \frac{2}{\gamma(\gamma-1)}\left[\sum_{i=1}^{n} \bar{p}_{i}(\xi) f^{r}\left(\xi_{i}\right)-\left(\sum_{i=1}^{n} \bar{p}_{i}(\xi) f\left(\xi_{i}\right)\right)^{\gamma}\right] \\
= & \lim _{\|T\| \rightarrow 0} \operatorname{Var}_{\gamma}(f(\xi), \bar{p}(\xi)) .
\end{aligned}
$$

By (14), we obtain

$$
\begin{aligned}
V_{r, s}(f, p) & =\left[\frac{\operatorname{Var}_{r}(f, p)}{\operatorname{Var}_{s}(f, p)}\right]^{\frac{1}{r-s}}=\lim _{\|T\| \rightarrow 0}\left[\frac{\operatorname{Var}_{r}(f(\xi), \bar{p}(\xi))}{\operatorname{Var}_{s}(f(\xi), \bar{p}(\xi))}\right]^{\frac{1}{r-s}} \\
& =\lim _{\|T\| \rightarrow 0} V_{r, s}(f(\xi), \bar{p}(\xi)) .
\end{aligned}
$$

By Lemma 2, we have

$$
\min \{f(\xi)\} \leq V_{r, s}(f(\xi), \bar{p}(\xi)) \leq \max \{f(\xi)\}
$$

From (15) and (16), we obtain

$$
\begin{aligned}
\min _{t \in \Omega}\{f(t)\} & =\lim _{\|T\| \rightarrow 0} \min \{f(\xi)\} \leq \lim _{\|T\| \rightarrow 0} V_{r, s}(f(\xi), \bar{p}(\xi)) \\
& =V_{r, s}(f, p) \leq \lim _{\|T\| \rightarrow 0} \max \{f(\xi)\}=\max _{t \in \Omega}\{f(t)\}
\end{aligned}
$$

This completes the proof of Theorem 1 .

Remark 3 By [21], if the function $\phi: I \rightarrow \mathbb{R}$ has the property that $\phi^{\prime \prime}: I \rightarrow \mathbb{R}$ is a continuous and convex function, then for any $x \in I^{n}$ and $p \in S^{n}$, we obtain

$$
\begin{aligned}
\phi^{\prime \prime}\left(V_{3,2}(x, p)\right) & \leq \frac{2[A(\phi(x), p)-\phi(A(x, p))]}{\operatorname{Var}_{2}(x, p)} \\
& \leq \frac{1}{3}\left\{\max _{1 \leq i \leq n}\left\{\phi^{\prime \prime}\left(x_{i}\right)\right\}+A\left(\phi^{\prime \prime}(x), p\right)+\phi^{\prime \prime}(A(x, p))\right\} .
\end{aligned}
$$

Thus, according to the proof of Theorem 1 , we may see that: If the function $f: \Omega \rightarrow(0, \infty)$ is continuous and the function $\phi: f(\Omega) \rightarrow \mathbb{R}$ has the property that $\phi^{\prime \prime}: f(\Omega) \rightarrow \mathbb{R}$ is a 
continuous convex function, then

$$
\begin{aligned}
\phi^{\prime \prime}\left(V_{3,2}(f, p)\right) & \leq \frac{2[E(\phi \circ f, p)-\phi(E(f, p))]}{\operatorname{Var}_{2}(f, p)} \\
& \leq \frac{1}{3}\left\{\max _{t \in \Omega}\left\{\phi^{\prime \prime}(f(t))\right\}+E\left(\phi^{\prime \prime} \circ f, p\right)+\phi^{\prime \prime}(E(f, p))\right\},
\end{aligned}
$$

where $\phi \circ f$ is the composite of $\phi$ and $f$. Therefore, the Dresher variance mean $V_{r, s}(f, p)$ has a wide mathematical background.

\section{Proof of Theorem 2}

In this section, we use the same notations as in the previous section. In addition, for fixed $\gamma, r, s \in \mathbb{R}$, if $x \in(0, \infty)^{n}$ and $p \in S^{n}$, then the $\gamma$-order power mean of $x$ with respect to $p$ (see, e.g., [1-9]) is defined by

$$
M^{[\gamma]}(x, p)= \begin{cases}{\left[A\left(x^{\gamma}, p\right)\right]^{\frac{1}{\gamma}},} & \gamma \neq 0, \\ \exp A(\ln x, p), & \gamma=0,\end{cases}
$$

and the two-parameter Dresher mean of $x$ (see $[10,11]$ ) with respect to $p$ is defined by

$$
D_{r, s}(x, p)= \begin{cases}{\left[\frac{A\left(x^{r}, p\right)}{A\left(x^{s}, p\right)}\right] \frac{1}{r-s},} & r \neq s, \\ \exp \left[\frac{A\left(x^{s} \ln x, p\right)}{A\left(x^{s}, p\right)}\right], & r=s .\end{cases}
$$

We have the following well-known power mean inequality [1-9]: If $\alpha<\beta$, then

$$
M^{[\alpha]}(x, p) \leq M^{[\beta]}(x, p) .
$$

We also have the following result (see [2], p.74, and [10, 11]).

Lemma 3 (Dresher inequality) If $x \in(0, \infty)^{n}, p \in S^{n}$ and $(r, s),\left(r^{*}, s^{*}\right) \in \mathbb{R}^{2}$, then the inequality

$$
D_{r, s}(x, p) \geq D_{r^{*}, s^{*}}(x, p)
$$

holds if and only if

$$
\max \{r, s\} \geq \max \left\{r^{*}, s^{*}\right\} \quad \text { and } \min \{r, s\} \geq \min \left\{r^{*}, s^{*}\right\} .
$$

Proof Indeed, if (20) hold, since $D_{r, s}(x, p)=D_{s, r}(x, p)$, we may assume that $r \geq r^{*}$ and $s \geq s^{*}$. By the power mean inequality, we have

$$
\begin{aligned}
D_{r, s}(x, p) & =M^{[r-s]}\left(x, \frac{x^{s} p}{A\left(x^{s}, p\right)}\right) \geq M^{\left[r^{*}-s\right]}\left(x, \frac{x^{s} p}{A\left(x^{s}, p\right)}\right) \\
& =M^{\left[s-r^{*}\right]}\left(x, \frac{x^{r^{*}} p}{A\left(x^{r^{*}}, p\right)}\right) \geq M^{\left[s^{*}-r^{*}\right]}\left(x, \frac{x^{r^{*}} p}{A\left(x^{r^{*}}, p\right)}\right) \\
& =D_{r^{*}, s^{*}}(x, p) .
\end{aligned}
$$

If (19) holds, by [2], p.74 and [10, 11], (20) hold. 
Lemma 4 Let $x \in(0, \infty)^{n}, p \in S^{n}$ and $(r, s),\left(r^{*}, s^{*}\right) \in \mathbb{R}^{2}$. If (20) holds, then

$$
V_{r, s}(x, p) \geq V_{r^{*}, s^{*}}(x, p) .
$$

Proof If $x$ is a constant $n$-vector, our assertion clearly holds. We may, therefore, assume that there exist $i, j \in N_{n}$ such that $x_{i} \neq x_{j}$. We may further assume that

$$
r(r-1) s(s-1)(r-s) \neq 0 .
$$

Let $G=\left\{\Delta \Phi_{1}, \Delta \Phi_{2}, \ldots, \Delta \Phi_{l}\right\}$ be a partition of $\Phi=\left\{\left(t_{1}, t_{2}\right) \in[0, \infty)^{2} \mid t_{1}+t_{2} \leq 1\right\}$. Let the area of each $\Delta \Phi_{i}$ be denoted by $\left|\Delta \Phi_{i}\right|$, and let

$$
\|G\|=\max _{1 \leq k \leq l x, y \in \Delta \Phi_{k}} \max _{1}\{\|x-y\|\}
$$

be the 'norm' of the partition, then for any $\left(\xi_{k, 1}, \xi_{k, 2}\right) \in \Delta \Phi_{k}$, we have

$$
\iint_{\Phi}\left[w_{i, j}\left(x, p, t_{1}, t_{2}\right)\right]^{r-2} d t_{1} d t_{2}=\lim _{\|G\| \rightarrow 0} \sum_{k=1}^{l}\left[w_{i, j}\left(x, p, \xi_{k, 1}, \xi_{k, 2}\right)\right]^{r-2}\left|\Delta \Phi_{k}\right|
$$

By (11), when $\gamma(\gamma-1) \neq 0$, we have

$$
\begin{aligned}
\operatorname{Var}_{\gamma}(x, p) & =2 \sum_{1 \leq i<j \leq n} p_{i} p_{j}\left(x_{i}-x_{j}\right)^{2} \iint_{\Phi}\left[w_{i, j}\left(x, p, t_{1}, t_{2}\right)\right]^{\gamma-2} d t_{1} d t_{2} \\
& =2 \sum_{1 \leq i<j \leq n} p_{i} p_{j}\left(x_{i}-x_{j}\right)^{2} \lim _{\|G\| \rightarrow 0} \sum_{k=1}^{l}\left[w_{i, j}\left(x, p, \xi_{k, 1}, \xi_{k, 2}\right)\right]^{\gamma-2}\left|\Delta \Phi_{k}\right| \\
& =\lim _{\|G\| \rightarrow 0}\left\{2 \sum_{1 \leq i<j \leq n} p_{i} p_{j}\left(x_{i}-x_{j}\right)^{2} \sum_{k=1}^{l}\left[w_{i, j}\left(x, p, \xi_{k, 1}, \xi_{k, 2}\right)\right]^{\gamma-2}\left|\Delta \Phi_{k}\right|\right\} \\
& =\lim _{\|G\| \rightarrow 0}\left\{\sum_{1 \leq i<j \leq n, 1 \leq k \leq l} 2 p_{i} p_{j}\left|\Delta \Phi_{k}\right|\left(x_{i}-x_{j}\right)^{2}\left[w_{i, j}\left(x, p, \xi_{k, 1}, \xi_{k, 2}\right)\right]^{\gamma-2}\right\} .
\end{aligned}
$$

By (21) and Lemma 3, we then see that

$$
\begin{aligned}
V_{r, s}(x, p) & =\left[\frac{\operatorname{Var}_{r}(x, p)}{\operatorname{Var}_{s}(x, p)}\right]^{\frac{1}{r-s}} \\
& =\lim _{\|G\| \rightarrow 0}\left\{\frac{\sum_{1 \leq i<j \leq n, 1 \leq k \leq l} p_{i} p_{j}\left|\Delta \Phi_{k}\right|\left(x_{i}-x_{j}\right)^{2}\left[w_{i, j}\left(x, p, \xi_{k, 1}, \xi_{k, 2}\right)\right]^{r-2}}{\sum_{1 \leq i<j \leq n, 1 \leq k \leq l} p_{i} p_{j}\left|\Delta \Phi_{k}\right|\left(x_{i}-x_{j}\right)^{2}\left[w_{i, j}\left(x, p, \xi_{k, 1}, \xi_{k, 2}\right)\right]^{s-2}}\right\}^{\frac{1}{(r-2)-(s-2)}} \\
& \geq \lim _{\|G\| \rightarrow 0}\left\{\frac{\sum_{1 \leq i<j \leq n, 1 \leq k \leq l} p_{i} p_{j}\left|\Delta \Phi_{k}\right|\left(x_{i}-x_{j}\right)^{2}\left[w_{i, j}\left(x, p, \xi_{k, 1}, \xi_{k, 2}\right)\right]^{r^{*}-2}}{\sum_{1 \leq i<j \leq n, 1 \leq k \leq l} p_{i} p_{j}\left|\Delta \Phi_{k}\right|\left(x_{i}-x_{j}\right)^{2}\left[w_{i, j}\left(x, p, \xi_{k, 1}, \xi_{k, 2}\right)\right]^{s^{*}-2}}\right\}^{\frac{1}{\left(r^{*}-2\right)-\left(s^{*}-2\right)}} \\
& =V_{r^{*}, s^{*}}(x, p) .
\end{aligned}
$$

This ends the proof.

We may now easily obtain the proof of Theorem 2 . 
Proof Indeed, by (20), (15) and Lemma 4, we get that

$$
V_{r, s}(f, p)=\lim _{\|T\| \rightarrow 0} V_{r, s}(f(\xi), \bar{p}(\xi)) \geq \lim _{\|T\| \rightarrow 0} V_{r^{*}, s^{*}}(f(\xi), \bar{p}(\xi))=V_{r^{*}, s^{*}}(f, p) .
$$

This completes the proof of Theorem 2 .

\section{Proof of Theorem 3}

In this section, we use the same notations as in the previous two sections. In addition, let $I_{n}=(1,1, \ldots, 1)$ be an $n$-vector and

$$
Q_{+}=\left\{\frac{s}{r} \mid r \in\{1,2,3, \ldots\}, s \in\{0,1,2, \ldots\}\right\}
$$

let $S_{n}$ be the $(n-1)$-dimensional simplex that

$$
S_{n}=\left\{x \in(0, \infty)^{n} \mid \sum_{i=1}^{n} x_{i}=n\right\}
$$

and let

$$
F_{n}(x)=\sum_{i=1}^{n} x_{i}^{\gamma} \ln x_{i}-\frac{1}{\gamma-1}\left(\sum_{i=1}^{n} x_{i}^{\gamma}-n\right)
$$

be defined on $S_{n}$.

Lemma 5 Let $\gamma \in(1, \infty)$. If $x$ is a relative extremum point of the function $F_{n}: S_{n} \rightarrow \mathbb{R}$, then there exist $k \in N_{n}$ and $u, v \in(0, n)$ such that

$$
k u+(n-k) v=n,
$$

and

$$
F_{n}(x)=k u^{k} \ln u+(n-k) v^{k} \ln v-\frac{1}{\gamma-1}\left[k u^{k}+(n-k) v^{k}-n\right] .
$$

Proof Consider the Lagrange function

$$
L(x)=F_{n}(x)+\mu\left(\sum_{i=1}^{n} x_{i}-n\right)
$$

with

$$
\frac{\partial L}{\partial x_{j}}=x_{j}^{\gamma-1}\left(\gamma \ln x_{j}+1\right)-\frac{\gamma}{\gamma-1} x_{j}^{\gamma-1}+\mu=x_{j}^{\gamma-1} \mathcal{L}\left(x_{j}\right)=0, \quad j=1,2, \ldots, n,
$$

where the function $\mathcal{L}:(0, n) \rightarrow \mathbb{R}$ is defined by

$$
\mathcal{L}(t)=\gamma \ln t+\mu t^{1-\gamma}-\frac{1}{\gamma-1}
$$


Then

$$
x_{j} \in(0, n), \quad \mathcal{L}\left(x_{j}\right)=0, \quad j=1,2, \ldots, n .
$$

Note that

$$
\mathcal{L}^{\prime}(t)=\frac{\gamma}{t}-\frac{\gamma-1}{t^{\gamma}} \mu=\frac{\gamma}{t^{\gamma}}\left(t^{\gamma-1}-\frac{\gamma-1}{\gamma} \mu\right) .
$$

Hence, the function $\mathcal{L}:(0, n) \rightarrow \mathbb{R}$ has at most one extreme point, and $\mathcal{L}(t)$ has at most two roots in $(0, n)$. By $(24)$, we have

$$
\left|\left\{x_{1}, x_{2}, \ldots, x_{n}\right\}\right| \leq 2,
$$

where $\left|\left\{x_{1}, x_{2}, \ldots, x_{n}\right\}\right|$ denotes the count of elements in the set $\left\{x_{1}, x_{2}, \ldots, x_{n}\right\}$. Since $F_{n}$ : $S_{n} \rightarrow \mathbb{R}$ is a symmetric function, we may assume that there exists $k \in N_{n}$ such that

$$
\begin{aligned}
& x_{1}=x_{2}=\cdots=x_{k}=u, \\
& x_{k+1}=x_{k+2}=\cdots=x_{n}=v .
\end{aligned}
$$

That is, (23) and (22) hold. The proof is complete.

Lemma 6 Let $\gamma \in(1, \infty)$. If $x$ is a relative extremum point of the function $F_{n}: S_{n} \rightarrow \mathbb{R}$, then

$$
F_{n}(x) \geq 0 .
$$

Proof By Lemma 5, there exist $k \in N_{n}$ and $u, v \in(0, n)$ such that (23) and (22) hold. If $u=v=1$, then (25) holds. We may, therefore, assume without loss of generality that $0<$ $u<1<v$. From (22), we see that

$$
\frac{k}{n}=\frac{1-v}{u-v}
$$

Putting (26) into (23), we obtain that

$$
\begin{aligned}
F_{n}(x) & =n\left\{\frac{k}{n} u^{\gamma} \ln u+\left(1-\frac{k}{n}\right) v^{\gamma} \ln v-\frac{1}{\gamma-1}\left[\frac{k}{n} u^{\gamma}+\left(1-\frac{k}{n}\right) v^{\gamma}-1\right]\right\} \\
& =n\left\{\frac{1-v}{u-v} u^{\gamma} \ln u+\frac{u-1}{u-v} v^{\gamma} \ln v-\frac{1}{\gamma-1}\left[\frac{1-v}{u-v} u^{\gamma}+\frac{u-1}{u-v} v^{\gamma}-1\right]\right\} \\
& =n\left\{\frac{1-v}{u-v} u^{\gamma} \ln u+\frac{u-1}{u-v} v^{\gamma} \ln v-\frac{1}{\gamma-1}\left[\frac{1-v}{u-v}\left(u^{\gamma}-1\right)+\frac{u-1}{u-v}\left(v^{\gamma}-1\right)\right]\right\} \\
& =\frac{n(1-v)(u-1)}{u-v}\left\{\frac{u^{\gamma} \ln u}{u-1}+\frac{v^{\gamma} \ln v}{1-v}-\frac{1}{\gamma-1}\left[\frac{u^{\gamma}-1}{u-1}+\frac{v^{\gamma}-1}{1-v}\right]\right\} \\
& =\frac{n(1-u)(v-1)}{v-u}\left\{\frac{v^{\gamma} \ln v}{v-1}-\frac{u^{\gamma} \ln u}{u-1}-\frac{1}{\gamma-1}\left[\frac{v^{\gamma}-1}{v-1}-\frac{u^{\gamma}-1}{u-1}\right]\right\} \\
& =\frac{n(1-u)(v-1)}{(\gamma-1)(v-u)}[\psi(v, \gamma)-\psi(u, \gamma)],
\end{aligned}
$$



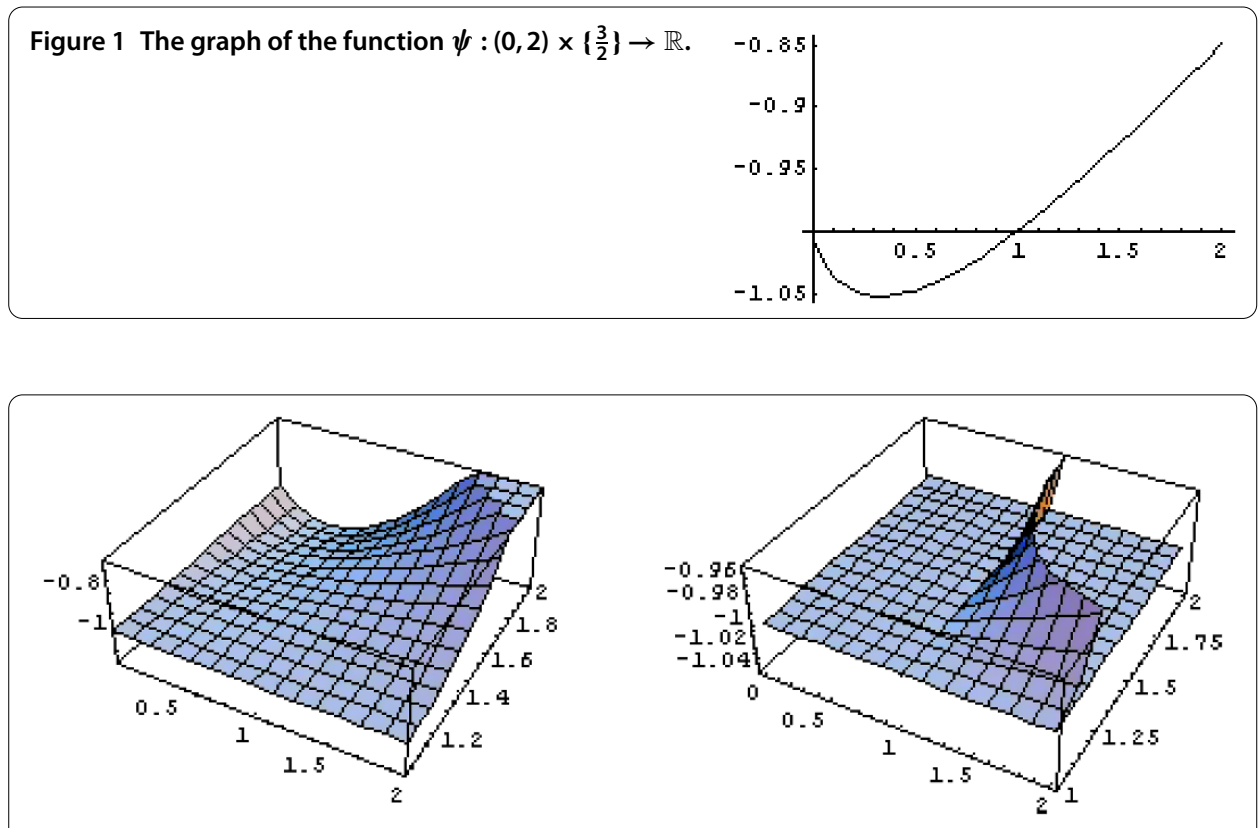

Figure 2 The graph of the function $\psi:(0,2) \times(1,2] \rightarrow \mathbb{R}$.

where the auxiliary function $\psi:(0, \infty) \times(1, \infty) \rightarrow \mathbb{R}$ is defined by

$$
\psi(t, \gamma)=\frac{(\gamma-1) t^{\gamma} \ln t-\left(t^{\gamma}-1\right)}{t-1}
$$

Since $\frac{n(1-u)(v-1)}{(\gamma-1)(v-u)}>0$, by (27), inequality (25) is equivalent to the following inequality:

$$
\psi(v, \gamma) \geq \psi(u, \gamma), \quad \forall u \in(0,1), \forall v \in(1, \infty), \forall \gamma \in(1, \infty)
$$

By the software Mathematica, we can depict the image of the function $\psi:(0,2) \times\left\{\frac{3}{2}\right\} \rightarrow \mathbb{R}$ in Figure 1, and the image of the function $\psi:(0,2) \times(1,2] \rightarrow \mathbb{R}$ in Figure 2 .

Now, let us prove the following inequalities:

$$
\begin{aligned}
& \psi(u, \gamma)<-1, \quad \forall u \in(0,1), \forall \gamma \in(1, \infty) \\
& \psi(v, \gamma)>-1, \quad \forall v \in(1, \infty), \forall \gamma \in(1, \infty) .
\end{aligned}
$$

By Cauchy's mean value theorem, there exists $\xi \in(u, 1)$ such that

$$
\begin{aligned}
\psi(u, \gamma) & =\frac{(\gamma-1) u^{\gamma} \ln u-\left(u^{\gamma}-1\right)}{u-1}=\frac{(\gamma-1) \ln u-1+u^{-\gamma}}{u^{1-\gamma}-u^{-\gamma}} \\
& =\left.\frac{\partial\left[(\gamma-1) \ln u-1+u^{-\gamma}\right] / \partial u}{\partial\left(u^{1-\gamma}-u^{-\gamma}\right) / \partial u}\right|_{u=\xi}=\frac{(\gamma-1) \xi^{-1}-\gamma \xi^{-\gamma-1}}{(1-\gamma) \xi^{-\gamma}+\gamma \xi^{-\gamma-1}} \\
& =\frac{(\gamma-1) \xi^{\gamma}-\gamma}{-(\gamma-1) \xi+\gamma}<\frac{(\gamma-1) \xi-\gamma}{-(\gamma-1) \xi+\gamma}=-1 .
\end{aligned}
$$

Therefore, inequality (29) holds. 
Next, note that

$$
\begin{aligned}
\psi(v, \gamma)>-1 & \Leftrightarrow \quad \frac{(\gamma-1) v^{\gamma} \ln v-\left(v^{\gamma}-1\right)}{v-1}>-1 \\
& \Leftrightarrow \quad(\gamma-1) v^{\gamma} \ln v-v^{\gamma}+v>0 \\
& \Leftrightarrow \quad \psi_{*}(v, \gamma)=(\gamma-1) \ln v-1+v^{1-\gamma}>0 .
\end{aligned}
$$

By Lagrange's mean value theorem, there exists $\xi_{*} \in(1, v)$ such that

$$
\begin{aligned}
\psi_{*}(\nu, \gamma) & =\psi_{*}(\nu, \gamma)-\psi_{*}(1, \gamma) \\
& =\left.(\nu-1) \frac{\partial \psi_{*}(\nu, \gamma)}{\partial v}\right|_{\nu=\xi_{*}} \\
& =(\nu-1)\left[(\gamma-1) \xi_{*}^{-1}+(1-\gamma) \xi_{*}^{-\gamma}\right] \\
& =(\gamma-1)(\nu-1) \xi_{*}^{-\gamma}\left(\xi_{*}^{\gamma-1}-1\right)>0 .
\end{aligned}
$$

Hence, (31) holds. It then follows that inequality (30) holds.

By inequalities (29) and (30), we may easily obtain inequality (28). This ends the proof of Lemma 6.

Lemma 7 If $\gamma \in(1, \infty)$, then for any $x \in S_{n}$, inequality (25) holds.

Proof We proceed by induction.

(A) Suppose $n=2$. By the well-known non-linear programming (maximum) principle and Lemma 6, we only need to prove that

$$
\lim _{x_{1} \rightarrow 0,\left(x_{1}, x_{2}\right) \in S_{2}} F_{2}\left(x_{1}, x_{2}\right) \geq 0 \quad \text { and } \quad \lim _{x_{2} \rightarrow 0,\left(x_{1}, x_{2}\right) \in S_{2}} F_{2}\left(x_{1}, x_{2}\right) \geq 0 .
$$

We show the first inequality, the second being similar.

Indeed, it follows from Lagrange's mean value theorem that there exists $\gamma_{*} \in(1, \gamma)$ such that

$$
\begin{aligned}
\lim _{x_{1} \rightarrow 0,\left(x_{1}, x_{2}\right) \in S_{2}} F_{2}\left(x_{1}, x_{2}\right) & =2^{\gamma} \ln 2-\frac{2^{\gamma}-2}{\gamma-1} \\
& =2^{\gamma} \frac{(\gamma-1) \ln 2-1+2^{1-\gamma}}{\gamma-1} \\
& =\left.2^{\gamma} \frac{d\left[(\gamma-1) \ln 2-1+2^{1-\gamma}\right]}{d \gamma}\right|_{\gamma=\gamma_{*}} \\
& =2^{\gamma} \ln 2\left(1-2^{1-\gamma_{*}}\right) \\
& >0 .
\end{aligned}
$$

(B) Assume by induction that the function $F_{n-1}: S_{n-1} \rightarrow \mathbb{R}$ satisfies $F_{n-1}(y) \geq 0$ for all $y \in S_{n-1}$. We prove inequality (25) as follows. By Lemma 6, we only need to prove that

$$
\lim _{x_{1} \rightarrow 0, x \in S_{n}} F_{n}(x) \geq 0, \quad \ldots, \quad \lim _{x_{n} \rightarrow 0, x \in S_{n}} F_{n}(x) \geq 0 .
$$


We will only show the last inequality. If we set

$$
y=\left(y_{1}, y_{2}, \ldots, y_{n-1}\right)=\frac{n-1}{n}\left(x_{1}, x_{2}, \ldots, x_{n-1}\right),
$$

then $y \in S_{n-1}$. By Lagrange's mean value theorem, there exists $\gamma_{*} \in(1, \gamma)$ such that

$$
\begin{aligned}
& \frac{1}{\gamma-1}\left[(\gamma-1)\left(\ln \frac{n}{n-1}\right)-1+\left(\frac{n}{n-1}\right)^{1-\gamma}\right] \\
& =\left.\frac{\partial}{\partial \gamma}\left[(\gamma-1)\left(\ln \frac{n}{n-1}\right)-1+\left(\frac{n}{n-1}\right)^{1-\gamma}\right]\right|_{\gamma=\gamma_{*}} \\
& =\left(\ln \frac{n}{n-1}\right)\left[1-\left(\frac{n}{n-1}\right)^{1-\gamma_{*}}\right] .
\end{aligned}
$$

Thus, by the power mean inequality

$$
\sum_{i=1}^{n-1} y_{i}^{\gamma} \geq(n-1)\left(\frac{1}{n-1} \sum_{i=1}^{n-1} y_{i}\right)^{\gamma}=n-1
$$

and induction hypothesis, we see that

$$
\begin{aligned}
\lim _{x_{n} \rightarrow 0, x \in S_{n}} F_{n}(x) & =\sum_{i=1}^{n-1} x_{i}^{\gamma} \ln x_{i}-\frac{1}{\gamma-1}\left(\sum_{i=1}^{n} x_{i}^{\gamma}-n\right) \\
= & \sum_{i=1}^{n-1}\left(\frac{n}{n-1} y_{i}\right)^{\gamma} \ln \left(\frac{n}{n-1} y_{i}\right)-\frac{1}{\gamma-1}\left[\sum_{i=1}^{n-1}\left(\frac{n}{n-1} y_{i}\right)^{\gamma}-n\right] \\
& =\left(\frac{n}{n-1}\right)^{\gamma}\left\{\left(\ln \frac{n}{n-1}\right) \sum_{i=1}^{n-1} y_{i}^{\gamma}+\sum_{i=1}^{n-1} y_{i}^{\gamma} \ln y_{i}-\frac{1}{\gamma-1}\left[\sum_{i=1}^{n-1} y_{i}^{\gamma}-n\left(\frac{n}{n-1}\right)^{\gamma}\right]\right\} \\
& =\left(\frac{n}{n-1}\right)^{\gamma}\left\{\left(\ln \frac{n}{n-1}\right) \sum_{i=1}^{n-1} y_{i}^{r}+F_{n-1}(y)-\frac{1}{\gamma-1}\left[n-1-n\left(\frac{n-1}{n}\right)^{\gamma}\right]\right\} \\
\geq & \left(\frac{n}{n-1}\right)^{\gamma}\left\{\left(\ln \frac{n}{n-1}\right)(n-1)-\frac{1}{\gamma-1}\left[n-1-n\left(\frac{n}{n-1}\right)^{\gamma}\right]\right\} \\
& =(n-1)\left(\frac{n}{n-1}\right)^{\gamma} \frac{1}{\gamma-1}\left[(\gamma-1)\left(\ln \frac{n}{n-1}\right)-1+\left(\frac{n-1}{n}\right)^{\gamma-1}\right] \\
& =(n-1)\left(\frac{n}{n-1}\right)^{\gamma} \frac{1}{\gamma-1}\left[(\gamma-1)\left(\ln \frac{n}{n-1}\right)-1+\left(\frac{n-1}{n}\right)^{1-\gamma}\right]
\end{aligned}
$$

This ends the proof of Lemma 7. 
Lemma 8 Let $x \in(0, \infty)^{n}$ and $p \in S^{n}$. If $r>s \geq 1$, then

$$
V_{r, s}(x, p) \geq\left(\frac{s}{r}\right)^{\frac{1}{r-s}} A(x, p),
$$

and the coefficient $\left(\frac{s}{r}\right)^{\frac{1}{r-s}}$ in (32) is the best constant.

Proof We may assume that there exist $i, j \in N_{n}$ such that $x_{i} \neq x_{j}$. By continuity considerations, we may also assume that $r>s>1$.

(A) Suppose $p=n^{-1} I_{n}$. Then (32) can be rewritten as

$$
V_{r, s}\left(x, n^{-1} I_{n}\right) \geq\left(\frac{s}{r}\right)^{\frac{1}{r-s}} A\left(x, n^{-1} I_{n}\right),
$$

or

$$
\left[\frac{\operatorname{Var}_{r}\left(x, n^{-1} I_{n}\right)}{\left.\operatorname{Var}_{s}\left(x, n^{-1} I_{n}\right)\right)}\right]^{\frac{1}{r-s}} \geq\left(\frac{s}{r}\right)^{\frac{1}{r-s}} A\left(x, n^{-1} I_{n}\right),
$$

or

$$
\left[\frac{s(s-1)}{r(r-1)} \frac{A\left(x^{r}, n^{-1} I_{n}\right)-A^{r}\left(x, n^{-1} I_{n}\right)}{A\left(x^{s}, n^{-1} I_{n}\right)-A^{s}\left(x, n^{-1} I_{n}\right)}\right]^{\frac{1}{r-s}} \geq\left(\frac{s}{r}\right)^{\frac{1}{r-s}} A\left(x, n^{-1} I_{n}\right) .
$$

That is,

$$
\mathcal{F}_{r}\left(x, n^{-1} I_{n}\right) \geq \mathcal{F}_{s}\left(x, n^{-1} I_{n}\right),
$$

where we have introduced the auxiliary function

$$
\mathcal{F}_{\gamma}(x, p)=\ln \frac{A\left(x^{\gamma}, p\right)-A^{\gamma}(x, p)}{(\gamma-1) A^{\gamma}(x, p)}, \quad \gamma>1
$$

Since, for any $t \in(0, \infty)$, we have $\mathcal{F}_{\gamma}\left(t x, n^{-1} I_{n}\right)=\mathcal{F}_{\gamma}\left(x, n^{-1} I_{n}\right)$, we may assume that $x \in S_{n}$. By Lemma 7, we have

$$
\begin{aligned}
\frac{\partial \mathcal{F}_{\gamma}\left(x, n^{-1} I_{n}\right)}{\partial \gamma} & =\frac{\partial}{\partial \gamma}\left[\ln \left(\frac{1}{n} \sum_{i=1}^{n} x_{i}^{\gamma}-1\right)-\ln (\gamma-1)\right] \\
& =\frac{n^{-1} \sum_{i=1}^{n} x_{i}^{\gamma} \ln x_{i}}{n^{-1} \sum_{i=1}^{n} x_{i}^{\gamma}-1}-\frac{1}{\gamma-1} \\
& =\frac{\sum_{i=1}^{n} x_{i}^{\gamma} \ln x_{i}-(\gamma-1)^{-1}\left(\sum_{i=1}^{n} x_{i}^{\gamma}-n\right)}{\sum_{i=1}^{n} x_{i}^{\gamma}-n} \\
& =\frac{F_{n}(x)}{\sum_{i=1}^{n} x_{i}^{\gamma}-n} \geq 0 .
\end{aligned}
$$

Hence, for a fixed $x \in(0, \infty)^{n}, \mathcal{F}_{\gamma}\left(x, n^{-1} I_{n}\right)$ is increasing with respect to $\gamma$ in $(1, \infty)$. Thus, by $r>s>1$, we obtain (33) and (32). 
(B) Suppose $p \neq n^{-1} I_{n}$, but $p \in Q_{+}^{n}$. Then there exists $N \in\{1,2,3, \ldots\}$ such that $N p_{i} \in$ $\{0,1,2, \ldots\}$ for $i=1, \ldots, n$. Setting

$$
x_{*}=(\underbrace{x_{1}, \ldots, x_{1}}_{N p_{1}}, \underbrace{x_{2}, \ldots, x_{2}}_{N p_{2}}, \ldots, \underbrace{x_{n}, \ldots, x_{n}}_{N p_{n}}), \quad N p_{1}+N p_{2}+\cdots+N p_{n}=m \text {, }
$$

and

$$
p_{*}=m^{-1} I_{m}
$$

then $x_{*} \in(0, \infty)^{m}, p_{*} \in S^{m}$. Inequality (32) can then be rewritten as

$$
V_{r, s}\left(x_{*}, p_{*}\right) \geq\left(\frac{s}{r}\right)^{\frac{1}{r-s}} A\left(x_{*}, p_{*}\right)
$$

According to the result in (A), inequality (34) holds.

(C) Suppose $p \neq n^{-1} I_{n}$ and $p \in S^{n} \backslash Q_{+}^{n}$. Then it is easy to see that there exists a sequence $\left\{p^{(k)}\right\}_{k=1}^{\infty} \subset Q_{+}^{n}$ such that $\lim _{k \rightarrow \infty} p^{(k)}=p$. According to the result in (B), we get

$$
V_{r, s}\left(x, p^{(k)}\right) \geq\left(\frac{s}{r}\right)^{\frac{1}{r-s}} A\left(x, p^{(k)}\right), \quad \forall k \in\{1,2,3, \ldots\} .
$$

Therefore

$$
V_{r, s}(x, p)=\lim _{k \rightarrow \infty} V_{r, s}\left(x, p^{(k)}\right) \geq\left(\frac{s}{r}\right)^{\frac{1}{r-s}} \lim _{k \rightarrow \infty} A\left(x, p^{(k)}\right)=\left(\frac{s}{r}\right)^{\frac{1}{r-s}} A(x, p)
$$

Next, we show that the coefficient $\left(\frac{s}{r}\right)^{\frac{1}{r-s}}$ is the best constant in (32). Assume that the inequality

$$
V_{r, s}(x, p) \geq C_{r, s} A(x, p)
$$

holds. Setting

$$
x=(0, \underbrace{1,1, \ldots, 1}_{n-1})
$$

and $p=n^{-1} I_{n}$ in (35), we obtain

$$
\begin{aligned}
& {\left[\frac{s(s-1)}{r(r-1)} \frac{\frac{n-1}{n}-\left(\frac{n-1}{n}\right)^{r}}{\frac{n-1}{n}-\left(\frac{n-1}{n}\right)^{s}}\right]^{\frac{1}{r-s}} \geq C_{r, s} \frac{n-1}{n}} \\
& \quad \Leftrightarrow \quad\left[\frac{s(s-1)}{r(r-1)} \frac{1-\left(\frac{n-1}{n}\right)^{r-1}}{1-\left(\frac{n-1}{n}\right)^{s-1}}\right]^{\frac{1}{r-s}} \geq C_{r, s} \frac{n-1}{n} .
\end{aligned}
$$

In (36), by letting $n \rightarrow \infty$, we obtain

$$
\left(\frac{s}{r}\right)^{\frac{1}{r-s}} \geq C_{r, s}
$$

Hence, the coefficient $\left(\frac{s}{r}\right)^{\frac{1}{r-s}}$ is the best constant in (32). The proof is complete. 
Remark 4 If $x \in(0, \infty)^{n}, p \in S^{n}$ and $r>s>1$, then there cannot be any $\theta, C_{r, s}: \theta \in(0, \infty)$ and $C_{r, s} \in(0, \infty)$ such that

$$
V_{r, s}(x, p) \leq C_{r, s} M^{[\theta]}(x, p) .
$$

Indeed, if there exist $\theta \in(0, \infty)$ and $C_{r, s} \in(0, \infty)$ such that (37) holds, then by setting

$$
x=(1, \underbrace{0,0, \ldots, 0}_{n-1}),
$$

and $p=n^{-1} I_{n}$ in (37), we see that

$$
\left[\frac{s(s-1)}{r(r-1)} \frac{1-n^{1-r}}{1-n^{1-s}}\right]^{\frac{1}{r-s}} \leq C_{r, s} n^{-\frac{1}{\theta}}
$$

which implies

$$
\left[\frac{s(s-1)}{r(r-1)}\right]^{\frac{1}{r-s}}=\lim _{n \rightarrow \infty}\left[\frac{s(s-1)}{r(r-1)} \frac{1-n^{1-r}}{1-n^{1-s}}\right]^{\frac{1}{r-s}} \leq \lim _{n \rightarrow \infty} C_{r, s} n^{-\frac{1}{\theta}}=0,
$$

which is a contradiction.

Remark 5 The method of the proof of Lemma 8 is referred to as the descending method in $[6,7,13,23-27]$, but the details in this paper are different.

We now return to the proof of Theorem 3 .

Proof By (15) and Lemma 8, we obtain

$$
V_{r, s}(f, p)=\lim _{\|T\| \rightarrow 0} V_{r, s}(f(\xi), \bar{p}(\xi)) \geq\left(\frac{s}{r}\right)^{\frac{1}{r-s}} \lim _{\|T\| \rightarrow 0} A(f(\xi), \bar{p}(\xi))=\left(\frac{s}{r}\right)^{\frac{1}{r-s}} E(f, p) .
$$

Thus, inequality (4) holds. Furthermore, by Lemma 8 , the coefficient $\left(\frac{s}{r}\right)^{\frac{1}{r-s}}$ is the best constant. This completes the proof of Theorem 3.

\section{Applications in space science}

It is well known that there are nine planets in the solar system, i.e., Mercury, Venus, Earth, Mars, Jupiter, Saturn, Uranus, Neptune and Pluto. In this paper, we also believe that Pluto is a planet in the solar system. In space science, we always consider the gravity to the Earth from other planets in the solar system (see Figure 3).

We can build the mathematical model of the problem. Let the masses of these planets be $m_{0}, m_{1}, \ldots, m_{n}$, where $m_{0}$ denotes the mass of the Earth and $1 \leq n \leq 8$. At moment $T_{0}$, in $\mathbb{R}^{3}$, let the coordinate of the center of the Earth be $o=(0,0,0)$ and the center of the $i$ th planet be $p_{i}=\left(p_{i 1}, p_{i 2}, p_{i 3}\right)$, and the distance between $p_{i}$ and $o$ be $\left\|p_{i}\right\|=\sqrt{p_{i 1}^{2}+p_{i 2}^{2}+p_{i 3}^{2}}$, where $i=1,2, \ldots, n$. By the famous law of gravitation, the gravity to the Earth $o$ from the planets $p_{1}, p_{2}, \ldots, p_{n}$ is

$$
F:=G_{0} m_{0} \sum_{i=1}^{n} \frac{m_{i} p_{i}}{\left\|p_{i}\right\|^{3}}
$$


Figure 3 The graph of the planet system $\operatorname{PS}\{P, m, B(g, r)\}_{\mathbb{R}^{3}}^{4}$.

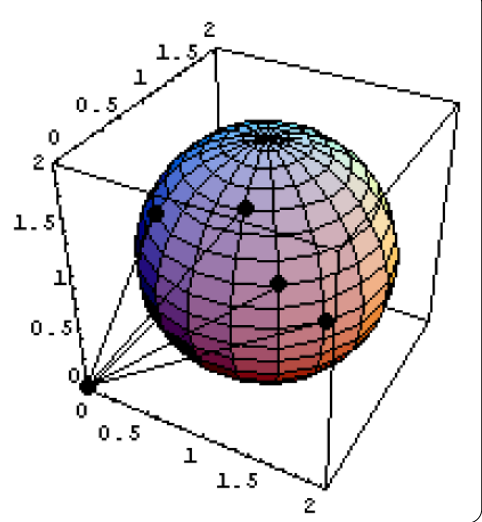

where $G_{0}$ is the gravitational constant in the solar system. Assume the coordinate of the center of the sun is $g=\left(g_{1}, g_{2}, g_{3}\right)$, then there exists a ball $\mathrm{B}(g, r)$ such that the planets $p_{1}, p_{2}, \ldots, p_{n}$ move in this ball. In other words, at any moment, we have

$$
p_{i} \in \mathrm{B}(g, r), \quad i=1,2, \ldots, n,
$$

where $r$ is the radius of the ball $\mathrm{B}(g, r)$.

We denote by $\theta_{i, j}:=\angle\left(p_{i}, p_{j}\right)$ the angle between the vectors $\overrightarrow{o p}_{i}$ and $\overrightarrow{o p}_{j}$, where $1 \leq i \neq$ $j \leq n$. This angle is also considered as the observation angle between two planets $p_{i}, p_{j}$ from the Earth $o$, which can be measured from the Earth by telescope.

Without loss of generality, we suppose that $n \geq 2, G_{0}=1, m_{0}=1$ and $\sum_{i=1}^{n} m_{i}=1$ in this paper.

We can generalize the above problem to an Euclidean space. Let $\mathbb{E}$ be an Euclidean space. For two vectors $\alpha \in \mathbb{E}$ and $\beta \in \mathbb{E}$, the inner product of $\alpha, \beta$ and the norm of $\alpha$ are denoted by $\langle\alpha, \beta\rangle$ and $\|\alpha\|=\sqrt{\langle\alpha, \alpha\rangle}$, respectively. The angle between $\alpha$ and $\beta$ is denoted by

$$
\angle(\alpha, \beta):=\arccos \frac{\langle\alpha, \beta\rangle}{\|\alpha\| \cdot\|\beta\|} \in[0, \pi],
$$

where $\alpha$ and $\beta$ are nonzero vectors.

Let $g \in \mathbb{E}$, we say the set

$$
\mathrm{B}(g, r):=\{x \in \mathbb{E} \mid\|x-g\| \leq r\}
$$

is a closed sphere and the set

$$
\mathrm{S}(g, r):=\{x \in \mathbb{E} \mid\|x-g\|=r\}
$$

is a spherical, where $r \in \mathbb{R}_{++}=(0,+\infty)$.

Now let us define the planet system and the $\lambda$-gravity function.

Let $\mathbb{E}$ be an Euclidean space, the dimension of $\mathbb{E} \operatorname{dim} \mathbb{E} \geq 3, P=\left(p_{1}, p_{2}, \ldots, p_{n}\right)$ and $m=$ $\left(m_{1}, m_{2}, \ldots, m_{n}\right)$ be the sequences of $\mathbb{E}$ and $\mathbb{R}_{++}$, respectively, and $\mathrm{B}(g, r)$ be a closed sphere in $\mathbb{E}$. The set

$$
\operatorname{PS}\{P, m, \mathrm{~B}(g, r)\}_{\mathbb{E}}^{n}:=\{P, m, \mathrm{~B}(g, r)\}
$$


is called the planet system if the following three conditions hold:

(H1) $\left\|p_{i}\right\|>0, i=1,2, \ldots, n$;

(H2) $p_{i} \in \mathrm{B}(g, r), i=1,2, \ldots, n$;

(H3) $\sum_{i=1}^{n} m_{i}=1$.

Let $\operatorname{PS}\{P, m, \mathrm{~B}(g, r)\}_{\mathbb{E}}^{n}$ be a planet system. The function

$$
F_{\lambda}: \mathbb{E}^{n} \rightarrow \mathbb{E}, F_{\lambda}(P)=\sum_{i=1}^{n} \frac{m_{i} p_{i}}{\left\|p_{i}\right\|^{\lambda+1}}
$$

is called a $\lambda$-gravity function of the planet system $\operatorname{PS}\{P, m, \mathrm{~B}(g, r)\}_{\mathbb{E}}^{n}$, and $F_{0}$ is the gravity kernel of $F_{\lambda}$, where $\lambda \in[0,+\infty)$.

Write

$$
p_{i}=\left\|p_{i}\right\| e_{i} ; \quad \theta_{i, j}:=\angle\left(p_{i}, p_{j}\right) \in[0, \pi] ; \quad \theta_{i}:=\angle\left(p_{i}, g\right) \in[0, \pi] .
$$

The matrix $A=\left[\left\langle e_{i}, e_{j}\right\rangle\right]_{n \times n}=\left[\cos \theta_{i, j}\right]_{n \times n}$ is called an observation matrix of the planet system $\operatorname{PS}\{P, m, \mathrm{~B}(g, r)\}_{\mathbb{E}}^{n}, \theta_{i, j}(j \neq i, 1 \leq i, j \leq n)$ and $\theta_{i}(1 \leq i \leq n)$ are called the observation angle and the center observation angle of the planet system, respectively. For the planet system, we always consider that the observation matrix $A$ is a constant matrix in this paper.

It is worth noting that the norm $\left\|F_{0}\right\|$ of the gravity kernel $F_{0}=F_{0}(P)$ is independent of $\left\|p_{1}\right\|,\left\|p_{2}\right\|, \ldots,\left\|p_{n}\right\| ;$ furthermore,

$$
0 \leq\left\|F_{0}\right\|=\sqrt{m A m^{T}} \leq 1,
$$

where $m^{T}$ is the transpose of the row vector $m$, and

$$
m A m^{T}=\sum_{1 \leq i, j \leq n} m_{i} m_{j} \cos \theta_{i, j}
$$

is a quadratic.

In fact, from $F_{0}=\sum_{i=1}^{n} m_{i} e_{i}$ and $\left\langle e_{i}, e_{j}\right\rangle=\cos \theta_{i j}$, we have that

$$
\begin{aligned}
& 0 \leq\left\|F_{0}\right\|=\sqrt{\left\langle F_{0}, F_{0}\right\rangle}=\sqrt{\sum_{1 \leq i, j \leq n} m_{i} m_{j}\left\langle e_{i}, e_{j}\right\rangle}=\sqrt{m A m^{T}} ; \\
& \left\|F_{0}\right\|=\left\|\sum_{i=1}^{n} m_{i} e_{i}\right\| \leq \sum_{i=1}^{n}\left\|m_{i} e_{i}\right\|=1 .
\end{aligned}
$$

Let $\mathrm{PS}\{P, m, \mathrm{~B}(g, 1)\}_{\mathbb{E}}^{n}$ be a planet system. By the above definitions, the gravity to the Earth $o$ from $n$ planets $p_{1}, p_{2}, \ldots, p_{n}$ in the solar system is $F_{2}(P)$, and $\left\|F_{2}(P)\right\|$ is the norm of $F_{2}(P)$. If we take a point $q_{i}$ in the ray $o p_{i}$ such that $\left\|\overrightarrow{o q}_{i}\right\|=1$, and place a planet at $q_{i}$ with mass $m_{i}$ for $i=1,2, \ldots, n$, then the gravity of these $n$ planets $q_{1}, q_{2}, \ldots, q_{n}$ to the Earth $o$ is $F_{0}(P)$.

Let $\operatorname{PS}\{P, m, \mathrm{~B}(g, 1)\}_{\mathbb{E}}^{n}$ be a planet system. If we believe that $g$ is a molecule and $p_{1}, p_{2}, \ldots, p_{n}$ are atoms of $g$, then the gravity to another atom $o$ from $n$ atoms $p_{1}, p_{2}, \ldots, p_{n}$ of $g$ is $F_{2}(P)$.

In the solar system, the gravity of $n$ planets $p_{1}, p_{2}, \ldots, p_{n}$ to the planet $o$ is $F_{2}(P)$, while for other galaxy in the universe, the gravity may be $F_{\lambda}(P)$, where $\lambda \in(0,2) \cup(2,+\infty)$. 
Let $\operatorname{PS}\{P, m, \mathrm{~B}(g, r)\}_{\mathbb{E}}^{n}$ be a planet system. Then the function

$$
f_{\lambda}: \mathbb{E}^{n} \rightarrow \mathbb{R}_{++}, f_{\lambda}(P)=\sum_{i=1}^{n} \frac{m_{i}}{\left\|p_{i}\right\|^{\lambda}}
$$

is called an absolute $\lambda$-gravity function of the planet system $\operatorname{PS}\{P, m, \mathrm{~B}(g, r)\}_{\mathbb{E}}^{n}$, where $\lambda \in$ $[0,+\infty)$.

Let $P$ be a planetary sequence in the solar system. Then $\frac{1}{n} f_{2}(P)$ is the average value of the gravities of the planets $p_{1}, p_{2}, \ldots, p_{n}$ to the Earth $o$.

Let $P$ be a planetary sequence in the solar system. If we think that $m_{i}$ is the radiation energy of the planet $p_{i}$, then, according to optical laws, the radiant energy received by the Earth $o$ is $c \frac{m_{i}}{\left\|p_{i}\right\|^{2}}, i=1,2, \ldots, n$, and the total radiation energy received by the Earth $o$ is $c f_{2}(P)$, where $c>0$ is a constant.

By Minkowski's inequality (see [28])

$$
\|x+y\| \leq\|x\|+\|y\|, \quad \forall x, y \in \mathbb{E},
$$

we know that if $F_{\lambda}(P)$ and $f_{\lambda}(P)$ are a $\lambda$-gravity function and an absolute $\lambda$-gravity function of the planet system $\operatorname{PS}\{P, m, \mathrm{~B}(g, r)\}_{\mathbb{E}}^{n}$, respectively, then we have

$$
\left\|F_{\lambda}(P)\right\| \leq f_{\lambda}(P), \quad \forall \lambda \in[0,+\infty) .
$$

Now, we will define absolute $\lambda$-gravity variance and $\lambda$-gravity variance. To this end, we need the following preliminaries.

Two vectors $\mathbf{x}$ and $\mathbf{y}$ in $\mathbb{E}$ are said to be in the same (opposite) direction if (i) $\mathbf{x}=0$ or $\mathbf{y}=0$, or (ii) $\mathbf{x} \neq 0$ and $\mathbf{y} \neq 0$ and $\mathbf{x}$ is a positive (respectively negative) constant multiple of $\mathbf{y}$. Two vectors $\mathbf{x}$ and $\mathbf{y}$ in the same (opposite) direction are indicated by $\mathbf{x} \uparrow \mathbf{y}$ (respectively $\mathbf{x} \downarrow \mathbf{y})$.

We say that the set $\mathbb{S}:=S(0,1)$ is a unit sphere in $\mathbb{E}$.

For each $\alpha \in \mathbb{S}$, we say that the set

$$
\Pi_{\alpha}:=\{\gamma \in \mathbb{E} \mid\langle\gamma, \boldsymbol{\alpha}\rangle=1\}
$$

is the tangent plane to the unit sphere $\mathbb{S}$ at the vector $\boldsymbol{\alpha}$. It is obvious that

$$
\boldsymbol{\gamma} \in \Pi_{\boldsymbol{\alpha}} \quad \Leftrightarrow \quad\langle\boldsymbol{\gamma}-\boldsymbol{\alpha}, \boldsymbol{\alpha}\rangle=0 \quad \Leftrightarrow \quad \boldsymbol{\alpha} \perp \boldsymbol{\gamma}-\boldsymbol{\alpha} .
$$

Assume that $\boldsymbol{\alpha}, \boldsymbol{\beta} \in \mathbb{S}$, and that $\boldsymbol{\alpha} \pm \boldsymbol{\beta} \neq 0$. We then say that the set

$$
\boldsymbol{\alpha} \boldsymbol{\beta}:=\left\{\digamma_{\alpha, \beta}(t) \mid t \in(-\infty, \infty)\right\},
$$

where

$$
\digamma_{\alpha, \beta}(t)=\frac{(1-t) \boldsymbol{\alpha}+t \boldsymbol{\beta}}{\|(1-t) \boldsymbol{\alpha}+t \boldsymbol{\beta}\|},
$$


are straight lines on the unit sphere $\mathbb{S}$, and that the sets

$$
\begin{array}{ll}
{[\boldsymbol{\alpha} \boldsymbol{\beta}]:=\left\{\digamma_{\alpha, \beta}(t) \mid \lambda \in[0,1]\right\}, \quad(\boldsymbol{\alpha} \boldsymbol{\beta}]:=\left\{\digamma_{\alpha, \beta}(t) \mid t \in(0,1]\right\},} \\
{[\boldsymbol{\alpha} \boldsymbol{\beta}):=\left\{\digamma_{\alpha, \beta}(t) \mid t \in[0,1)\right\}, \quad(\boldsymbol{\alpha} \boldsymbol{\beta}):=\left\{\digamma_{\alpha, \beta}(t) \mid t \in(0,1)\right\},}
\end{array}
$$

are the straight line segments on the sphere $\mathbb{S}$, and that $\|\boldsymbol{\alpha} \boldsymbol{\beta}\|:=\angle(\boldsymbol{\alpha}, \boldsymbol{\beta})=\arccos \langle\boldsymbol{\alpha}, \boldsymbol{\beta}\rangle$ is the length of these line segments.

It is easy to see that $\boldsymbol{\alpha}+\boldsymbol{\beta} \neq 0$ implies $(1-t) \boldsymbol{\alpha}+t \boldsymbol{\beta} \neq 0$. Thus, we may easily get the existence and uniqueness of these line segments. Similarly, $\boldsymbol{\alpha} \pm \boldsymbol{\beta} \neq 0$ implies that $\|\boldsymbol{\alpha} \boldsymbol{\beta}\| \in$ $(0, \pi)$.

Assuming that $\gamma \in \Pi_{\alpha} \cap \Pi_{\beta}$, and that $\boldsymbol{\alpha}, \boldsymbol{\beta}, \boldsymbol{\gamma}$ are linearly dependent vectors, we say that $\boldsymbol{\gamma}-\boldsymbol{\alpha}$ is the tangent vector to the line segment $[\boldsymbol{\alpha} \boldsymbol{\beta})$ at $\boldsymbol{\alpha}$. By definition, we see that there exist $u, v \in \mathbb{R}$ such that

$$
\boldsymbol{\gamma}=u \boldsymbol{\alpha}+v \boldsymbol{\beta}
$$

Therefore

$$
\begin{aligned}
& 1=\langle\boldsymbol{\gamma}, \boldsymbol{\alpha}\rangle=u\langle\boldsymbol{\alpha}, \boldsymbol{\alpha}\rangle+v\langle\boldsymbol{\alpha}, \boldsymbol{\beta}\rangle=u+v\langle\boldsymbol{\alpha}, \boldsymbol{\beta}\rangle, \\
& 1=\langle\boldsymbol{\gamma}, \boldsymbol{\beta}\rangle=u\langle\boldsymbol{\alpha}, \boldsymbol{\beta}\rangle+v\langle\boldsymbol{\beta}, \boldsymbol{\beta}\rangle=u\langle\boldsymbol{\alpha}, \boldsymbol{\beta}\rangle+v .
\end{aligned}
$$

We infer from $\langle\boldsymbol{\alpha}, \boldsymbol{\beta}\rangle \in(-1,1)$ that

$$
u=v=\frac{1}{1+\langle\boldsymbol{\alpha}, \boldsymbol{\beta}\rangle}, \quad \gamma=\frac{\boldsymbol{\alpha}+\boldsymbol{\beta}}{1+\langle\boldsymbol{\alpha}, \boldsymbol{\beta}\rangle}, \quad \frac{\boldsymbol{\gamma}-\boldsymbol{\alpha}}{\|\boldsymbol{\gamma}-\boldsymbol{\alpha}\|}=\frac{\boldsymbol{\beta}-\langle\boldsymbol{\alpha}, \boldsymbol{\beta}\rangle \boldsymbol{\alpha}}{\|\boldsymbol{\beta}-\langle\boldsymbol{\alpha}, \boldsymbol{\beta}\rangle \boldsymbol{\alpha}\|}
$$

We define also the tangent vector of $[\boldsymbol{\alpha} \boldsymbol{\beta})$ at $\boldsymbol{\alpha}$ by $\boldsymbol{\beta}-\langle\boldsymbol{\alpha}, \boldsymbol{\beta}\rangle \boldsymbol{\alpha}$. The tangent vector $\boldsymbol{\beta}-$ $\langle\boldsymbol{\alpha}, \boldsymbol{\beta}\rangle \boldsymbol{\alpha}$ enjoys the following properties: If $\boldsymbol{\gamma} \in(\boldsymbol{\alpha} \boldsymbol{\beta})$, then

$$
(\boldsymbol{\gamma}-\langle\boldsymbol{\alpha}, \boldsymbol{\gamma}\rangle \boldsymbol{\alpha}) \uparrow(\boldsymbol{\beta}-\langle\boldsymbol{\alpha}, \boldsymbol{\beta}\rangle \boldsymbol{\alpha})
$$

In fact, there exists $t \in(0,1)$ such that

$$
\boldsymbol{\gamma}=\digamma_{\alpha, \beta}(t)=\frac{(1-t) \boldsymbol{\alpha}+t \boldsymbol{\beta}}{\|(1-t) \boldsymbol{\alpha}+t \boldsymbol{\beta}\|}
$$

Since $\langle\boldsymbol{\alpha}, \boldsymbol{\alpha}\rangle=1$, we see that

$$
\begin{aligned}
(\boldsymbol{\gamma}-\langle\boldsymbol{\alpha}, \boldsymbol{\gamma}\rangle \boldsymbol{\alpha}) & =\frac{(1-t) \boldsymbol{\alpha}+t \boldsymbol{\beta}}{\|(1-t) \boldsymbol{\alpha}+t \boldsymbol{\beta}\|}-\left\langle\boldsymbol{\alpha}, \frac{(1-t) \boldsymbol{\alpha}+t \boldsymbol{\beta}}{\|(1-t) \boldsymbol{\alpha}+t \boldsymbol{\beta}\|}\right\rangle \boldsymbol{\alpha} \\
& =\frac{(1-t) \boldsymbol{\alpha}+t \boldsymbol{\beta}-\langle\boldsymbol{\alpha},(1-t) \boldsymbol{\alpha}+t \boldsymbol{\beta}\rangle \boldsymbol{\alpha}}{\|(1-\lambda) \boldsymbol{\alpha}+t \boldsymbol{\beta}\|} \\
& \uparrow[(1-t) \boldsymbol{\alpha}+t \boldsymbol{\beta}-\langle\boldsymbol{\alpha},(1-t) \boldsymbol{\alpha}+t \boldsymbol{\beta}\rangle \boldsymbol{\alpha}] \\
& =t(\boldsymbol{\beta}-\langle\boldsymbol{\alpha}, \boldsymbol{\beta}\rangle \boldsymbol{\alpha}) \\
& \uparrow(\boldsymbol{\beta}-\langle\boldsymbol{\alpha}, \boldsymbol{\beta}\rangle \boldsymbol{\alpha}) .
\end{aligned}
$$


The angle between two line segments $[\boldsymbol{\alpha} \boldsymbol{\beta}),[\boldsymbol{\alpha} \boldsymbol{\gamma})$ on the unit sphere $\mathbb{S}$ is defined as

$$
\angle([\boldsymbol{\alpha} \boldsymbol{\beta}),[\boldsymbol{\alpha} \boldsymbol{\gamma})):=\angle(\boldsymbol{\beta}-\langle\boldsymbol{\alpha}, \boldsymbol{\beta}\rangle \boldsymbol{\alpha}, \boldsymbol{\gamma}-\langle\boldsymbol{\alpha}, \boldsymbol{\gamma}\rangle \boldsymbol{\alpha}) .
$$

If $[\boldsymbol{\alpha} \boldsymbol{\beta}),[\boldsymbol{\beta} \gamma)$, $[\boldsymbol{\gamma} \boldsymbol{\alpha})$ are three straight line segments on the unit sphere $\mathbb{S}$, and $\boldsymbol{\alpha} \notin \boldsymbol{\beta} \boldsymbol{\gamma}, \boldsymbol{\beta} \notin$ $\boldsymbol{\gamma} \boldsymbol{\alpha}, \boldsymbol{\gamma} \notin \boldsymbol{\alpha} \boldsymbol{\beta}$, then we say that the set $\Delta \boldsymbol{\alpha} \boldsymbol{\beta} \boldsymbol{\gamma}:=[\boldsymbol{\alpha} \boldsymbol{\beta}] \cup[\boldsymbol{\beta} \boldsymbol{\gamma}] \cup[\boldsymbol{\gamma} \boldsymbol{\alpha})$ is a spherical triangle. Write $A=\angle([\boldsymbol{\alpha} \boldsymbol{\beta}),[\boldsymbol{\alpha} \boldsymbol{\gamma})), B=\angle([\boldsymbol{\beta} \boldsymbol{\gamma}),[\boldsymbol{\beta} \boldsymbol{\alpha})), C=\angle([\boldsymbol{\gamma} \boldsymbol{\alpha}),[\boldsymbol{\gamma} \boldsymbol{\beta})), a=\|\boldsymbol{\beta} \boldsymbol{\gamma}\|, b=\|\boldsymbol{\gamma} \boldsymbol{\alpha}\|, c=$ $\|\boldsymbol{\alpha} \boldsymbol{\beta}\|$. Then we obtain that

$$
\begin{aligned}
\cos A & =\cos \angle(\boldsymbol{\beta}-\langle\boldsymbol{\alpha}, \boldsymbol{\beta}\rangle \boldsymbol{\alpha}, \boldsymbol{\gamma}-\langle\boldsymbol{\alpha}, \boldsymbol{\gamma}\rangle \boldsymbol{\alpha}) \\
& =\frac{\langle\boldsymbol{\beta}-\langle\boldsymbol{\alpha}, \boldsymbol{\beta}\rangle \boldsymbol{\alpha}, \boldsymbol{\gamma}-\langle\boldsymbol{\alpha}, \boldsymbol{\gamma}\rangle \boldsymbol{\alpha}\rangle}{\|\boldsymbol{\beta}-\langle\boldsymbol{\alpha}, \boldsymbol{\beta}\rangle \boldsymbol{\alpha}\| \cdot\|\boldsymbol{\gamma}-\langle\boldsymbol{\alpha}, \boldsymbol{\gamma}\rangle \boldsymbol{\alpha}\|} \\
& =\frac{\langle\boldsymbol{\beta}, \boldsymbol{\gamma}\rangle-\langle\boldsymbol{\alpha}, \boldsymbol{\beta}\rangle\langle\boldsymbol{\alpha}, \boldsymbol{\gamma}\rangle}{\sqrt{1-\langle\boldsymbol{\alpha}, \boldsymbol{\beta}\rangle^{2}} \cdot \sqrt{1-\langle\boldsymbol{\alpha}, \boldsymbol{\gamma}\rangle^{2}}} \\
& =\frac{\cos a-\cos b \cos c}{\sin b \sin c} .
\end{aligned}
$$

Thus, we may get the law of cosine for spherical triangle

$$
\cos a=\cos b \cos c+\sin b \sin c \cos A
$$

By (42) we may get the law of cosine for spherical triangle

$$
\cos A=-\cos B \cos C+\sin B \sin C \cos a .
$$

By (42) we may get the law of sine for spherical triangle

$$
\frac{\sin a}{\sin A}=\frac{\sin b}{\sin B}=\frac{\sin c}{\sin C}
$$

By $\cos A>-1, \cos a<1$ and (42)-(43), we get

$$
a, b, c \in(0, \pi), \quad b+c>a,
$$

or

$$
\angle(\boldsymbol{\beta}, \boldsymbol{\gamma})<\angle(\boldsymbol{\gamma}, \boldsymbol{\alpha})+\angle(\boldsymbol{\alpha}, \boldsymbol{\beta})
$$

and

$$
A, B, C \in(0, \pi), \quad A+B+C>\pi .
$$

Lemma 9 Let $\mathbb{E}$ be an Euclidean space, let the dimension of $\mathbb{E}$ satisfy $\operatorname{dim} \mathbb{E} \geq 3$, and let $\mathrm{B}(g, 1)$ be a closed sphere in $\mathbb{E}$. If $\|g\|>1$, then

$$
\max _{\boldsymbol{\alpha}, \boldsymbol{\beta} \in \mathrm{B}(g, 1)}\{\angle(\boldsymbol{\alpha}, \boldsymbol{\beta})\}=2 \arcsin \frac{1}{\|g\|} .
$$


Proof From $\alpha \in \mathrm{B}(g, 1)$ we get

$$
\begin{aligned}
& \langle\boldsymbol{\alpha}-g, \boldsymbol{\alpha}-g\rangle \leq 1, \\
& \|\boldsymbol{\alpha}\|^{2}-2\langle g, \boldsymbol{\alpha}\rangle+\|g\|^{2} \leq 1, \\
& \cos \angle(\boldsymbol{\alpha}, g)=\frac{\langle g, \boldsymbol{\alpha}\rangle}{\|\boldsymbol{\alpha}\|\|g\|} \geq \frac{\|\boldsymbol{\alpha}\|^{2}+\|g\|^{2}-1}{2\|\boldsymbol{\alpha}\|\|g\|} \geq \frac{\sqrt{\|g\|^{2}-1}}{\|g\|} .
\end{aligned}
$$

Thus,

$$
\angle(\boldsymbol{\alpha}, g) \leq \arcsin \frac{1}{\|g\|}
$$

Similarly, from $\beta \in \mathrm{B}(g, 1)$ we have

$$
\angle(\boldsymbol{\beta}, g) \leq \arcsin \frac{1}{\|g\|}
$$

If we set

$$
\boldsymbol{\alpha}^{*}=\frac{\boldsymbol{\alpha}}{\|\boldsymbol{\alpha}\|}, \quad \boldsymbol{\beta}^{*}=\frac{\boldsymbol{\beta}}{\|\boldsymbol{\beta}\|}, \quad g^{*}=\frac{g}{\|g\|},
$$

then $\boldsymbol{\alpha}^{*}, \boldsymbol{\alpha}^{*}, g^{*} \in \mathbb{S}$. According to inequalities (48), (49) and (45), we get

$$
\angle(\boldsymbol{\alpha}, \boldsymbol{\beta})=\angle\left(\boldsymbol{\alpha}^{*}, \boldsymbol{\beta}^{*}\right) \leq \angle\left(\boldsymbol{\alpha}^{*}, g^{*}\right)+\angle\left(g^{*}, \boldsymbol{\beta}^{*}\right)=\angle(\boldsymbol{\alpha}, g)+\angle(g, \boldsymbol{\beta}) \leq 2 \arcsin \frac{1}{\|g\|},
$$

hence

$$
\angle(\boldsymbol{\alpha}, \boldsymbol{\beta}) \leq 2 \arcsin \frac{1}{\|g\|}
$$

Now we discuss the conditions such that the equality in inequality (50) holds. From the above analysis, we know that these conditions are:

(a) $\boldsymbol{\alpha} \in \mathrm{B}(g, 1)$ and $\|\boldsymbol{\alpha}\|=\sqrt{\|g\|^{2}-1}$;

(b) $\boldsymbol{\beta} \in \mathrm{B}(g, 1)$ and $\|\boldsymbol{\beta}\|=\sqrt{\|g\|^{2}-1}$;

(c) $g^{*} \in\left[\boldsymbol{\alpha}^{*} \boldsymbol{\beta}^{*}\right]$ and $\angle\left(\boldsymbol{\alpha}^{*}, g^{*}\right)=\angle\left(g^{*}, \boldsymbol{\beta}^{*}\right)=\arcsin \frac{1}{\|g\|}$.

From (a) and (b) we know that the condition (c) can be rewritten as

(c) $\frac{g}{\|g\|}=\frac{\boldsymbol{\alpha}+\boldsymbol{\beta}}{\|\boldsymbol{\alpha}+\boldsymbol{\beta}\|}$ or $g \uparrow \boldsymbol{\alpha}+\boldsymbol{\beta}$.

Based on the above analysis, we know that the equality in inequality (50) can hold. Therefore, equality (47) holds. The lemma is proved.

It is worth noting that if $\operatorname{PS}\{P, m, \mathrm{~B}(g, 1)\}_{\mathbb{E}}^{n}$ is a planet system and $\|g\| \geq \sqrt{2}$, according to Lemma 9 and $p_{i}, p_{j} \in \mathrm{B}(g, 1)$, then we have that

$$
0 \leq \theta_{i, j}=\angle\left(p_{i}, p_{j}\right) \leq \max _{\boldsymbol{\alpha}, \boldsymbol{\beta} \in \mathrm{B}(g, 1)}\{\angle(\boldsymbol{\alpha}, \boldsymbol{\beta})\}=2 \arcsin \frac{1}{\|g\|} \leq \frac{\pi}{2}
$$


for any $i, j=1,2, \ldots, n$, and

$$
\begin{aligned}
& \left\|F_{\lambda}(P)\right\|^{2}=\sum_{1 \leq i, j \leq 1} m_{i} m_{j} \cos \theta_{i, j}\left(\frac{1}{\left\|p_{i}\right\|^{2}\left\|p_{j}\right\|^{2}}\right)^{\frac{\lambda}{2}}, \\
& \left\|F_{2}(P)\right\|^{\lambda}=\left[\sum_{1 \leq i, j \leq 1} m_{i} m_{j} \cos \theta_{i, j}\left(\frac{1}{\left\|p_{i}\right\|^{2}\left\|p_{j}\right\|^{2}}\right)\right]^{\frac{\lambda}{2}},
\end{aligned}
$$

where

$$
m_{i} m_{j} \cos \theta_{i, j} \geq 0, \quad i, j=1,2, \ldots, n
$$

Now, we will define absolute $\lambda$-gravity variance and $\lambda$-gravity variance.

Let $\operatorname{PS}\{P, m, \mathrm{~B}(g, r)\}_{\mathbb{E}}^{n}$ be a planet system, and let the functions $f_{\lambda}(P), F_{\lambda}(P)$ be an absolute $\lambda$-gravity function and a $\lambda$-gravity function of the planet system, respectively. We say the functions

$$
\operatorname{Var}_{\lambda}(P)=\operatorname{Var}_{\frac{\lambda}{2}}\left(p^{-2}, m\right)=\frac{8}{\lambda(\lambda-2)}\left[f_{\lambda}(P)-f_{2}^{\frac{\lambda}{2}}(P)\right], \quad 0<\lambda \neq 2,
$$

and

$$
\operatorname{Var}_{\lambda}^{*}(P)=\frac{8}{\lambda(\lambda-2)}\left[\left(\frac{\left\|F_{\lambda}(P)\right\|}{\left\|F_{0}(P)\right\|}\right)^{2}-\left(\frac{\left\|F_{2}(P)\right\|}{\left\|F_{0}(P)\right\|}\right)^{\lambda}\right], \quad 0<\lambda \neq 2,
$$

are absolute $\lambda$-gravity variance and $\lambda$-gravity variance of the planet system, respectively, where

$$
p=\left(\left\|p_{1}\right\|,\left\|p_{2}\right\|, \ldots,\left\|p_{n}\right\|\right), \quad p^{-2}=\left(\left\|p_{1}\right\|^{-2},\left\|p_{2}\right\|^{-2}, \ldots,\left\|p_{n}\right\|^{-2}\right)
$$

Let $\operatorname{PS}\{P, m, \mathrm{~B}(g, 1)\}_{\mathbb{E}}^{n}$ be a planet system, and $0<\lambda, \mu \neq 2, \lambda \neq \mu$. By Lemma 2 , we have

$$
\frac{1}{\max _{1 \leq i \leq n}\left\{\left\|p_{i}\right\|^{2}\right\}} \leq\left[\frac{\operatorname{Var}_{\lambda}(P)}{\operatorname{Var}_{\mu}(P)}\right]^{\frac{2}{\lambda-\mu}} \leq \frac{1}{\min _{1 \leq i \leq n}\left\{\left\|p_{i}\right\|^{2}\right\}}
$$

If $\|g\| \geq \sqrt{2}$, according to (51)-(54) and Lemma 2, we have

$$
\frac{1}{\max _{1 \leq i \leq n}\left\{\left\|p_{i}\right\|^{4}\right\}} \leq\left[\frac{\operatorname{Var}_{\lambda}^{*}(P)}{\operatorname{Var}_{\mu}^{*}(P)}\right]^{\frac{2}{\lambda-\mu}} \leq \frac{1}{\min _{1 \leq i \leq n}\left\{\left\|p_{i}\right\|^{4}\right\}} .
$$

Let $P$ be a planetary sequence in the solar system, and the gravity of the planet $p_{i}$ to the Earth $o$ is $F\left(p_{i}\right), i=1,2, \ldots, n$. Then inequalities (55) and (56) can be rewritten as

$$
\min _{1 \leq i \leq n}\left\{\frac{\left\|F\left(p_{i}\right)\right\|}{m_{i}}\right\} \leq\left[\frac{\operatorname{Var}_{\lambda}(P)}{\operatorname{Var}_{\mu}(P)}\right]^{\frac{2}{\lambda-\mu}} \leq \max _{1 \leq i \leq n}\left\{\frac{\left\|F\left(p_{i}\right)\right\|}{m_{i}}\right\}
$$

and

$$
\min _{1 \leq i \leq n}\left\{\frac{\left\|F\left(p_{i}\right)\right\|}{m_{i}}\right\} \leq\left[\frac{\operatorname{Var}_{\lambda}^{*}(P)}{\operatorname{Var}_{\mu}^{*}(P)}\right]^{\frac{1}{\lambda-\mu}} \leq \max _{1 \leq i \leq n}\left\{\frac{\left\|F\left(p_{i}\right)\right\|}{m_{i}}\right\},
$$

respectively. 
Let $\operatorname{PS}\{P, m, \mathrm{~B}(g, 1)\}_{\mathbb{E}}^{n}$ be a planet system. By Lemma 8 , if $\lambda>\mu>2$, then

$$
\frac{\operatorname{Var}_{\lambda}(P)}{\operatorname{Var}_{\mu}(P)} \geq \frac{\mu}{\lambda}\left[f_{2}(P)\right]^{\frac{\lambda-\mu}{2}} .
$$

If $\lambda>\mu>2$ and $\|g\| \geq \sqrt{2}$, according to (51)-(54) and Lemma 8, we have

$$
\frac{\operatorname{Var}_{\lambda}^{*}(P)}{\operatorname{Var}_{\mu}^{*}(P)} \geq \frac{\mu}{\lambda}\left[\frac{\left\|F_{2}(P)\right\|}{\left\|F_{0}(P)\right\|}\right]^{\lambda-\mu}
$$

where the coefficient $\mu / \lambda$ is the best constant in (59) and (60).

Remark 6 For some new literature related to space science, please see [4] and [25].

Competing interests

The authors declare that they have no competing interests.

Authors' contributions

All authors contributed equally and significantly in this paper. All authors read and approved the final manuscript.

\section{Author details}

${ }^{1}$ Institute of Mathematical Inequalities and Applications, Chengdu University, Chengdu, Sichuan 610106, P.R. China.

${ }^{2}$ Department of Mathematics, Tsing Hua University, Hsinchu, 30043, Taiwan.

\section{Acknowledgements}

This work is supported by the Natural Science Foundation of China (No. 10671136) and the Natural Science Foundation of Sichuan Province Education Department (No. 07ZA207).

\section{Received: 17 March 2013 Accepted: 18 July 2013 Published: 5 August 2013}

\section{References}

1. Bullen, PS, Mitrinnović, DS, Vasić, PM: Means and Their Inequalities. Reidel, Dordrecht (1988)

2. Kuang, JC: Applied Inequalities. Shandong Sci. \& Tech. Press, Jinan (2004) (in Chinese)

3. Mitrinović, DS, Pečarić, JE, Fink, AM: Classic and New Inequalities in Analysis. Kluwer Academic, Dordrecht (1993)

4. Wen, JJ, Gao, CB: Geometric inequalities involving the central distance of the centered 2-surround system. Acta Math. Sin. 51(4), 815-832 (2008) (in Chinese)

5. Wang, WL, Wen, JJ, Shi, HN: On the optimal values for inequalities involving power means. Acta Math. Sin. 47(6), 1053-1062 (2004) (in Chinese)

6. Wen, JJ, Wang, WL: The optimization for the inequalities of power means. J. Inequal. Appl.. 2006, Article ID 46782 (2006). doi:10.1155/JIA/2006/46782

7. Pečarić, JE, Wen, JJ, Wang, WL, Lu, T: A generalization of Maclaurin's inequalities and its applications. Math. Inequal. Appl. 8(4), 583-598 (2005)

8. Wen, JJ, Cheng, SS, Gao, CB: Optimal sublinear inequalities involving geometric and power means. Math. Bohem. 134(2), 133-149 (2009)

9. Ku, HT, Ku, MC, Zhang, XM: Generalized power means and interpolating inequalities. Proc. Am. Math. Soc. 127(1), 145-154 (1999)

10. Páles, Z: Inequalities for sums of powers. J. Math. Anal. Appl. 131(1), 265-270 (1988)

11. Bjelica, M: Asymptotic planarity of Dresher mean values. Mat. Vesn. 57, 61-63 (2005)

12. Wen, JJ, Han, TY, Gao, CB: Convergence tests on constant Dirichlet series. Comput. Math. Appl. 62(9), 3472-3489 (2011)

13. Yang, H, Wen, JJ, Wang, WL: The method of descending dimension for establishing inequalities (II). Sichuan Daxue Xuebao 44(4), 753-758 (2007)

14. Dresher, M: Moment spaces and inequalities. Duke Math. J. 20, 261-271 (1953)

15. Daskin, JM: Dresher's inequality. Am. Math. Mon. 49, 687-688 (1952)

16. Beckenbach, EF, Bellman, R: Inequalities, 3rd edn. Springer, Berlin (1971)

17. Brenner, JL, Carlson, BC: Homogenous mean values: weights and asymptotes. J. Math. Anal. Appl. 123, 265-280 (1987)

18. $\mathrm{Hu}, \mathrm{K}:$ Problems in Analytic Inequality. Wuhan University Press, Wuhan (2003) (in Chinese)

19. Wen, JJ, Zhang, ZH: Jensen type inequalities involving homogeneous polynomials. J. Inequal. Appl. 2010, Article ID $850215(2010)$

20. Pečarić, JE, Svrtan, D: New refinements of the Jensen inequalities based on samples with repetitions. J. Math. Anal. Appl. 222, 365-373 (1998)

21. Wen, JJ: The inequalities involving Jensen functions. J. Syst. Sci. Math. Sci. 27(2), 208-218 (2007) (in Chinese)

22. Gao, CB, Wen, Jj: Inequalities of Jensen-Pečarić-Svrtan-Fan type. J. Inequal. Pure Appl. Math. 9, Article ID 74 (2008)

23. Timofte, V: On the positivity of symmetric polynomial functions. J. Math. Anal. Appl. 284, 174-190 (2003) 
24. Wen, JJ, Yuan, J, Yuan, SF: An optimal version of an inequality involving the third symmetric means. Proc. Indian Acad. Sci. Math. Sci. 118(4), 505-516 (2008)

25. Gao, CB, Wen, JJ: Theory of surround system and associated inequalities. Comput. Math. Appl. 63, 1621-1640 (2012)

26. Wen, JJ, Wang, WL: Chebyshev type inequalities involving permanents and their application. Linear Algebra Appl. 422(1), 295-303 (2007)

27. Wen, JJ, Wang, WL: The inequalities involving generalized interpolation polynomial. Comput. Math. Appl. 56(4), 1045-1058 (2008)

28. Gardner, RJ: The Brunn-Minkowski inequality. Bull., New Ser., Am. Math. Soc. 39, 355-405 (2002)

doi:10.1186/1029-242X-2013-366

Cite this article as: Wen et al.: Inequalities involving Dresher variance mean. Journal of Inequalities and Applications $20132013: 366$

Submit your manuscript to a SpringerOpen ${ }^{\circ}$ journal and benefit from:

- Convenient online submission

- Rigorous peer review

Immediate publication on acceptance

- Open access: articles freely available online

- High visibility within the field

- Retaining the copyright to your article

Submit your next manuscript at $>$ springeropen.com 